Research Article

\title{
Diagnosis of the Misaligned Faults of the Vertical Test Instrument of High-Precision Industrial Robot Reducer
}

\author{
Zhen Yu $\mathbb{D D}^{1}$ and Yuan Zhang $\mathbb{D}^{2}$ \\ ${ }^{1}$ State Key Laboratory of Precision Measuring Technology and Instrument, Tianjin University, Tianjin 300072, China \\ ${ }^{2}$ School of Management, China University of Mining \& Technology, Beijing, China \\ Correspondence should be addressed to Yuan Zhang; zhangyuan_yuan999@163.com
}

Received 20 April 2021; Revised 23 June 2021; Accepted 16 July 2021; Published 27 July 2021

Academic Editor: Francisco Beltran-Carbajal

Copyright (c) 2021 Zhen Yu and Yuan Zhang. This is an open access article distributed under the Creative Commons Attribution License, which permits unrestricted use, distribution, and reproduction in any medium, provided the original work is properly cited.

\begin{abstract}
High-precision reducer is the core component of industrial robots. In order to achieve the comprehensive performance testing of precision reducers, an instrument with a vertical layout and a cylindrical structure is designed. As a rotating machine, the inevitable coupling misalignment of the instrument can lead to vibration faults which lead to errors in the test. So it is pretty necessary to diagnose and monitor the coupling misalignment while the instrument is working. The causes of the coupling misaligned fault of the instrument and the relationship between the misalignment fault and torque ripple are analyzed in this paper. A method of using the torque transducer in the measurement chain of the instrument to diagnose the coupling misalignment is proposed in this paper. Many experiments have been done to test the capability of detecting the coupling misalignment using this method. Experimental results show that the amplitude of torque ripple of the shaft is linearly related to the coupling misalignment and is quadratically related to the rotation speed of the shaft when the misalignment exists in the shaft. The combination of components at the rotation frequency (fr) and the additional components can be used to diagnose faults due to coupling misalignment.
\end{abstract}

\section{Introduction}

As a core component of the actuators in robots, the robot reducer directly affects their performance factors, such as the kinematic accuracy, load capacity, and fatigue life [1]. Therefore, the comprehensive performance test of precision reducers is required for developing high-precision industrial robots, and it has become the focus of attention of researchers [2]. Some progress has been achieved concerning the performance testing of precision reducers $[3,4]$. The reducer detector often shows vibration faults caused by shaft misalignment [5]. An instrument, designed by Dhaouadi and Ghorbel, is designed to be a vertical structure to ensure an excellent shaft misalignment [6-8]. However, the methods to reduce the shaft misalignment caused by the manufacturing tolerances incur high costs and require much effort [9]. Even if an accurate alignment is secured, it cannot be continued for a long duration due to many effects, such as thermal deformation and load imbalance [10, 11]. Many studies [12-14] indicated that the misalignment would undoubtedly lead to deterioration of whole lubrication characteristics of bearing and even the failure of the system.

Flexible couplings are usually used to overcome slight misalignment of the rotating machinery [15-17]. However, as is described above, many factors can increase misaligned faults. Therefore, a feasible method for misaligned fault diagnosis is required. Recently, many efforts have been made to diagnose misaligned faults of rotating machinery through the combination of signal acquisition, data processing, and fault classification [18-22]. Vibration analysis is the most common technique for monitoring and diagnosing mechanical systems [23-25]. The signal acquisition for certain mechanical faults is accomplished by installing an accelerometer or proximity probe $[26,27]$. However, due to the confusing spectral characteristics of vibration, vibrationbased misalignment detection led to less reliability [10]. 
Furthermore, especially for the vertical reducer detector with a torque sensor in the measuring chain, the additional sensors mounted on the mechanism can cause a series of problems such as signals acquired to vary according to the positioning of sensors [28, 29], onefold information acquisition [30], and additional cost.

Some scholars and experts have made some progress on the research of detection and monitoring of coupling misalignment based on torque signal [31]. However, their work only shows the possibilities of torque measurements as a valuable technique for detecting misalignment, and more theoretical studies are needed to show the relationship between the coupling misalignment and the ripple of torque. In other works, techniques for estimating torque from the sensing of the electrical variables of the motors have been proposed and tested using the frequency spectrum of the current electrical motor for diagnosis [16]. However, the estimated torque, which includes the component due to either misalignment of stator and rotor or rotor eccentricity of the motor, cannot represent the torque transmitted by the shaft. Thus, it cannot show the effect of coupling misalignment of the shaft clearly. Besides, the experiment is carried out on the horizontal instrument, which cannot represent the effect of the method on the vertical instrument.

In order to solve the problem of diagnosis coupling misalignment in the vertical robot reducer detector, a method of using the torque transducer to diagnose the coupling misaligned fault of performance testing instruments of precision reducers is proposed in this paper to overcome the shortcoming of diagnosis of misaligned fault using an accelerometer or proximity probe. The paper is mainly organized as follows: Firstly, the structure of a vertical performance testing instrument of precision reducers, including guide rail mechanism, measurement module on the input side (MMIS), tested assemblies (TA), measurement module on the output side (MMOS), and workbench, is introduced in Section 2 combined with analysis of the causes of the misaligned fault. Next, the relationships between the misaligned fault and the torque ripple are analyzed in Section 3. Moreover, the experiment's effects using the Fast Fourier Transform and power spectrum analysis of the torque signal to diagnose the misaligned fault are revealed in Section 4. Finally, some conclusions are given in the fifth section.

\section{Structure of the Vertical Instrument and Analysis of the Causes of the Misaligned Fault}

The vertical instrument is designed to measure the comprehensive performance of the reducer, as shown in Figure 1. This instrument consists of four assistance modules, which are guide rail mechanism, tested assemblies (TA), power supply system, and workbench, and two measurement modules, namely, the measurement module at the input side of the reducer (MMIS) and that at the output side of the reducer (MMOS). Each measurement module is mainly composed of the five following subsystems: motor, torque transducer, test mode conversion part, angle encoder, and other connecting parts. The red component in Figure 1 represents the TA. The robot reducer was installed inside the TA, which is used to achieve a rapid installation of different reducer types. The connections with the measurement systems adopt a uniform and standard structure.

The instrument is of vertical type in which functional components are sequentially connected in series in the vertical direction. MMIS, MMOS, and TA are the core components. The causes of the misaligned fault are as follows:

(1) Although the disk supports inside the hollow cylinder are designed to ensure that the shafts are centered, the manufacturing tolerances and assembly error of the disk supports can still lead to shaft misalignment.

(2) The flexible coupling is used in the connecting parts to overcome slight misalignment between the shafts. However, many factors can increase misaligned faults, and flexible coupling can never solve the problem caused by increasing misalignment.

(3) Moreover, the test mode conversion components allow the input or output shaft of the tested reducer to be under unconstrained, driven, or locked conditions, thereby satisfying the functional requirements of different dynamic and static performance tests. However, there is no guarantee that the conversion components move along the axis. The changing of the test mode can increase the misaligned faults because of the motion of the conversion components.

Since all the three factors above can lead to the misaligned fault and the misalignment mainly between the shafts connected by the flexible coupling and spline coupling, a feasible method is required to realize the misaligned fault diagnosis. In this paper, the torque transducer is used to diagnose the misaligned fault of performance testing instruments of precision reducers through testing the torque ripple. The relationships between the misaligned fault and the torque ripple are analyzed in the next section.

\section{Relationships between the Misaligned Fault and the Torque Ripple}

There are three common types of shaft misalignment (as shown in Figure 2): parallel misalignment (a), angle misalignment (b), and comprehensive misalignment (c). Due to the inevitable manufacturing tolerances and assembly error, the three kinds of misalignment faults shown in Figure 2 can exist in the flexible coupling and spline coupling at each of the two shafts of MMIS and MMOS. Especially for the rectangular spline sleeve, which connects two spline shafts at the same time, the misalignment fault can exist. Due to the excessive spline clearance, both spline shafts can be considered unconstrained at one end, and the other end is supported in the state of high-precision bearing. In the transmission process, it is likely that the misalignment between the two spline shafts and the spline sleeve changes with the rotation of the shaft. That is, the three states in Figure 2 may appear alternately. 


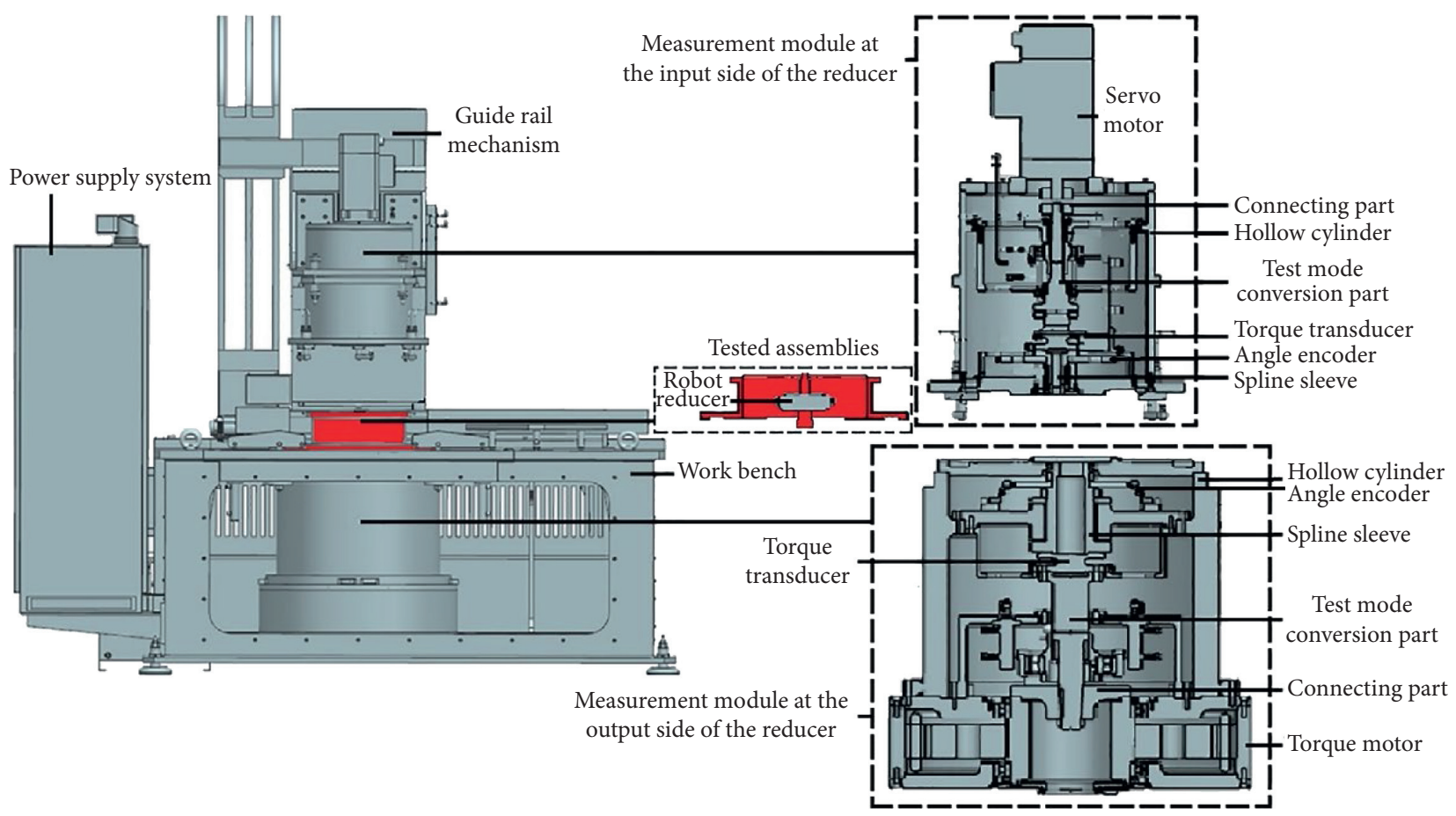

FIgURE 1: The vertical precision robot reducer detector.

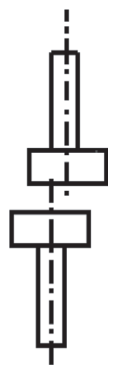

(a)

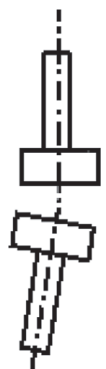

(b)

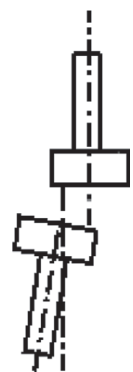

(c)

Figure 2: Three states of shaft misalignment. (a) The parallel misalignment; (b) the angle misalignment; (c) the comprehensive misalignment.

The rotating shaft of MMIS, which is the same as that of MMOS, is shown in Figure 3. When the parallel or angular offsets exist in the shaft, the mass center of the shaft does not coincide with the rotation center. The shaft will be affected by the electromagnetic torque of the motor and the eccentric torque. Under the effect of the cogging effect and misalignments, the electromagnetic torque of the motor and the eccentric torque change with the rotation of the shaft. The driving torque, which is defined as the sum of the electromagnetic torque of the motor and the eccentric torque, can be expressed as the sum of averaged torque and torque ripple [32].

$$
T=\bar{T}+\sum_{n} T_{n} \cos \left(n \theta_{\text {out }}+\varnothing_{n}\right)
$$

where $\bar{T}$ and $T_{n}$ are the averaged torque and amplitude for the $n$th driving torque, respectively, $n$ is the harmonic orders of the output shaft, and $\theta_{\text {out }}$ and $\varnothing_{n}$ are the position of the output shaft and phase offset for the $n$th driving torque, respectively.

The ripple of the driving torque can cause the friction torque of the shaft to change with the shaft rotation. That is because the friction torque is caused by the friction generated in the bearing because of rotation. The ripple of the driving torque leads to the ripple of the acceleration of the shaft. The ripple of the acceleration of the shaft can cause the ripple of the rotation speed and finally leads to the ripple of the friction torque of bearings [33]. Then, the friction torque can be expressed as follows:

$$
T_{f}=\mu N r+\mu r m \Delta r \sum_{n} \omega_{n}^{2} \cos \left(n \theta_{\text {out }}+\varnothing_{n}\right),
$$

where $T_{f}$ and $\mu$ are the friction torque and friction coefficient, respectively, $N$ is the supporting force of the bearing, $r$ is the radius of the shaft, $m$ is the eccentric mass of the shaft, $\Delta r$ is the misalignment of the shaft, $n$ is the harmonic orders 


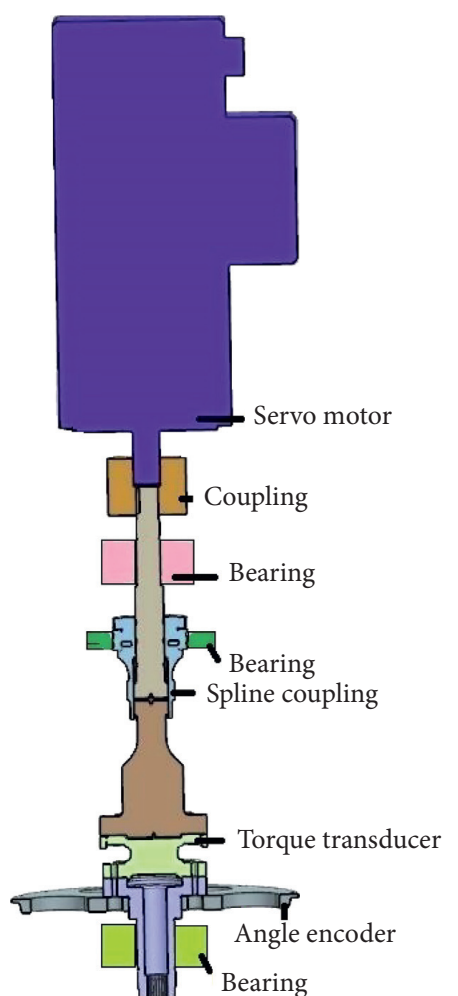

Figure 3: The rotating shaft of MMIS.

of the output shaft, $\omega_{n}$ is the amplitude for the $n$th rotation speed of the shaft, and $\theta_{\text {out }}$ and $\varnothing_{n}$ are the position of the output shaft and phase offset for the $n$th driving torque, respectively.

The driving torque and the friction torque, which are changing with the shaft rotation, eventually lead to the torque ripple of the shaft. As is analyzed above, the driving torque and the friction torque are affected by the misalignment. The torque ripple of the shaft is also affected by the misalignment. According to the analysis in the second section, the misalignment is mainly between the shafts connected by the flexible coupling and spline coupling. The impact of misalignment of various shaft parts on torque ripple, especially about the misalignment between the two spline shafts and the spline sleeve, is analyzed in detail in the following.

\subsection{Torque Ripple Caused by the Misalignment of Flexible} Coupling. When the parallel misalignment $\Delta x$ exists between the motor shaft and the transmission shaft, the flexible coupling connecting the two shafts will be deformed. According to Hooke's law, the deformed flexible coupling will produce reaction forces $F_{1}^{\prime}$ and $F_{2}^{\prime}$ on the shafts at both ends of the flexible coupling. Besides, the mass center of the shaft does not coincide with the rotation center. The shaft will be affected by the eccentric force. At this time, the transmission shaft is not only affected by the driving torque $T$ transmitted by the flexible coupling but also affected by the reaction force and the eccentric force caused by the parallel misalignment. The reaction force and the eccentric force will eventually be balanced by the supporting forces $F_{1}$ and $F_{2}$ provided by the bearing. Finally, the friction torque $T_{f 1}$ will be brought due to the reaction force and the eccentric force acting on the bearing. Therefore, the parallel misalignment $\Delta x$ exists between the motor shaft and drive shaft producing friction torque, which affects the torque transmitted through the shaft. Because the driving torque is changing with the shaft rotation, it will cause the rotation speed to change with the shaft rotation. The ripple of the rotation speed will lead to the friction torque change with the shaft rotation. Figure 4 shows the force and friction torque caused by the parallel misalignment $\Delta x$ between the motor shaft and the transmission shaft.

The torque transmitted by the transmission shaft $T_{1}$ is the drive torque minus the friction torque. When the parallel misalignment $\Delta x$ exists between the motor shaft and the transmission shaft, the torque transmitted by shaft $T^{\prime}$ can be expressed as follows [34]:

$$
\begin{aligned}
T_{1}= & \bar{T}+\sum_{n} T_{n} \cos \left(n \theta_{\text {out }}+\varnothing_{n}\right)-\mu_{1} k_{1} r_{1} \Delta x \\
& -\mu_{1} m_{1} r_{1} \Delta x \sum_{n} \omega_{n}^{2} \cos \left(n \theta_{\text {out }}+\varnothing_{n}\right),
\end{aligned}
$$

where $\overline{\bar{T}}$ and $T_{n}$ are the averaged torque and amplitude for the $n$th driving torque, respectively, $n$ is the harmonic orders of the output shaft, $\theta_{\text {out }}$ and $\varnothing_{n}$ are the position of the output shaft and phase offset for the $n$th driving torque, respectively, $\mu_{1}$ is the friction coefficient of the transmission shaft, $k_{1}$ is the coefficient of elasticity of the transmission shaft, $m_{1}$ is the mass of the transmission shaft, $r_{1}$ is the radius of the transmission shaft, $\Delta x$ is the parallel misalignment, and $\omega_{n}$ is the amplitude for the $n$th rotation speed of the shaft.

The misalignment errors comprise radial and angular displacements. When the angle misalignment $\Delta \theta$ exists between the motor shaft and the transmission shaft, the flexible coupling connecting the two shafts will be deformed. The deformed coupling will produce a reaction force on the shafts at both ends of the flexible coupling. According to Hooke's law, the reaction force on the transmission shaft caused by the angle misalignment can be expressed as follows:

$$
F=k_{1} L_{1} \sin (\Delta \theta)
$$

where $L_{1}$ is the length of the transmission shaft, $\Delta \theta$ is the angular misalignment, and $k_{1}$ is the coefficient of elasticity of the transmission shaft.

Besides, when the angular offsets exist in the shaft, the mass center of the shaft does not coincide with the rotation center. The shaft will be affected by the eccentric force. The eccentric force on the shaft caused by the angle misalignment can be expressed as follows:

$$
F_{e}=m_{1} L_{1} \sin (\Delta \theta) \sum_{n} \omega_{n}^{2} \cos \left(n \theta_{\text {out }}+\varnothing_{n}\right),
$$

where $F_{e}$ is the eccentric force, $m_{1}$ is the mass of the transmission shaft, $L_{1}$ is the length of the transmission shaft, $\Delta \theta$ is the angular misalignment, $n$ is the harmonic orders of the output shaft, $\omega_{n}$ is the amplitude for the $n$th rotation 


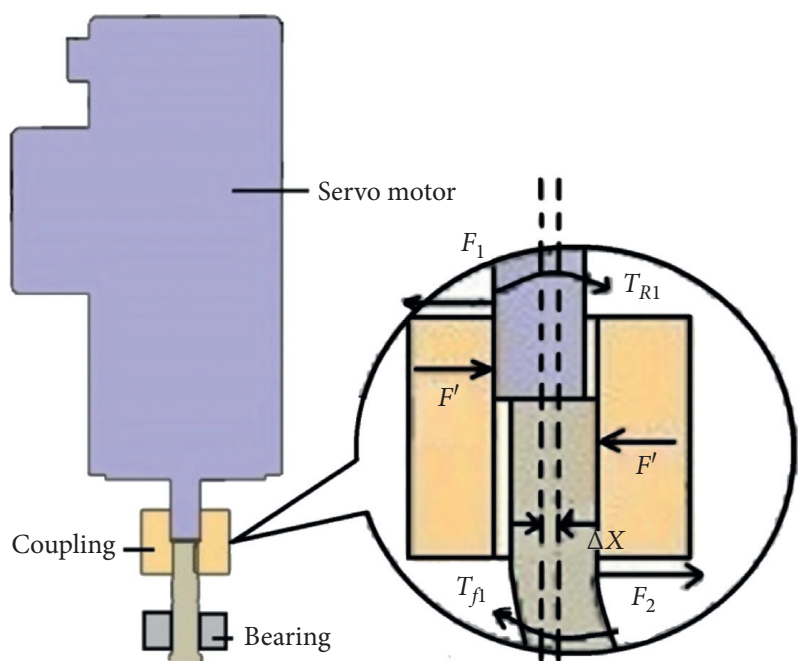

FIGURE 4: The force and the friction torque caused by the parallel misalignment of the flexible coupling.

speed of the shaft, and $\theta_{\text {out }}$ and $\varnothing_{n}$ are the position of the output shaft and phase offset for the $n$th driving torque, respectively.

At this time, the transmission shaft is not only affected by the driving torque $T$ transmitted by the flexible coupling but also affected by the reaction force and the eccentric force caused by the angular misalignment. The reaction force and the eccentric force will eventually be balanced by the supporting force provided by the bearing. Finally, the friction torque $T_{f 1}^{\prime}$ will be brought due to the reaction force and the eccentric force acting on the bearing. Therefore, the angle misalignment $\Delta \theta$ exists between the motor shaft and drive shaft producing friction torque, which affects the torque transmitted through the shaft. Because the driving torque is changing with the shaft rotation, it will cause the rotation speed to change with the shaft rotation. The ripple of the rotation speed will lead to the friction torque change with the shaft rotation. Figure 5 shows the force and friction torque caused by the angle misalignment $\Delta \theta$ between the motor shaft and the transmission shaft.

When an angular misalignment error exists, the torque transmitted by shaft $T_{1}^{\prime}$ is the drive torque minus the friction torque. When the angle misalignment $\Delta \theta$ exists between the motor shaft and the transmission shaft, the torque transmitted by shaft $T_{1}^{\prime}$ can be expressed as follows [35]:

$$
\begin{aligned}
T_{1}^{\prime}= & \bar{T}+\sum_{n} T_{n} \cos \left(n \theta_{\text {out }}+\varnothing_{n}\right)-\mu_{1} k_{1} L_{1} \sin (\Delta \theta) \\
& -\mu_{1} m_{1} r_{1} L_{1} \tan (\Delta \theta) \sum_{n} \omega_{n}^{2} \cos \left(n \theta_{\text {out }}+\varnothing_{n}\right),
\end{aligned}
$$

where $\bar{T}$ and $T_{n}$ are the averaged torque and amplitude for the $n$th driving torque, respectively, $L_{1}$ is the length of the transmitted shaft, $\Delta \theta$ is the angular misalignment, $k_{1}$ is the coefficient of elasticity of the transmission shaft, $\mu_{1}$ is the friction coefficient of the transmission shaft, $m_{1}$ is the mass of the transmission shaft, $r_{1}$ is the radius of the transmission shaft, $n$ is the harmonic orders of the output shaft, $\omega_{n}$ is the amplitude for the $n$th rotation speed of the shaft, and $\theta_{\text {out }}$

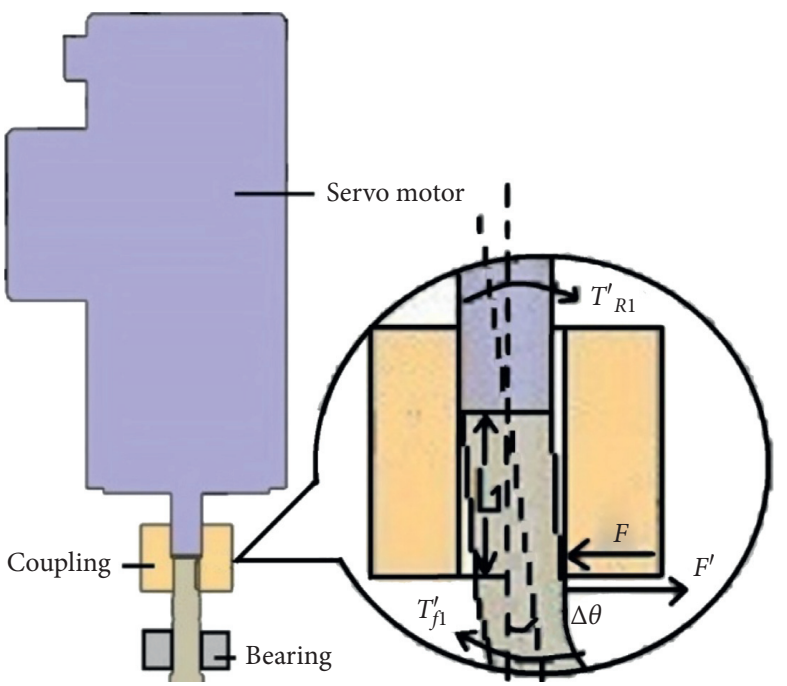

FIGURE 5: The force and the friction torque caused by the angular misalignment of the flexible coupling.

and $\varnothing_{n}$ are the position of the output shaft and phase offset for the $n$th driving torque, respectively.

\subsection{Torque Ripple Caused by the Misalignment of Spline} Coupling. The spline coupling is a complex structure. The spline structure in our instrument is equivalent to two sets of spline coupling. One spline sleeve connects two spline shafts at the same time. In general, the torque is transmitted to the spline sleeve through the transmission shaft and then is transferred to the torque transducer shaft by the spline sleeve. Due to the excessive spline clearance, both spline shafts can be considered unconstrained at one end and supported by a high-precision bearing at the other end. In the transmission process, it is likely that the misalignment between the two spline shafts and the spline sleeve changes with the rotation of the shaft. Besides, as long as there is a misalignment between the spline shaft and spline sleeve, only $25 \% \sim 50 \%$ of the spline's teeth participate in meshing simultaneously in actual work. Besides, the meshing of the spline shaft and spline sleeve is uneven, some of the spline's teeth meshing is tight, and some of the spline's teeth meshing is loose. As a result, the engagement force of each spline's teeth is different, and a resultant force is generated at the teeth of the spline shaft offset. The resultant force produces sliding between the spline sleeve and the spline shaft. So the friction torque, which affects the torque transmitted through the shaft, is generated. The resultant force is balanced by the friction force and support force provided by the bearing. Due to misalignment, the spline sleeve point meshed with the spline shaft at the spline shaft offset is changed with the shaft rotation.

When the parallel misalignment $\Delta x /$ exists between the transmission shaft and the torque transducer shaft, the meshing of the spline shaft and spline sleeve is uneven. The friction torque $T_{f 2}$ caused by the uneven meshing of the spline shaft and spline sleeve and the sliding between the spline sleeve and the spline shaft is generated. At this time, the torque transducer shaft is not only affected by the driving torque $T$ transmitted by the transmission shaft but also 
affected by the friction torque $T_{f 2}$ caused by the parallel misalignment. Therefore, the parallel misalignment $\Delta x /$ that exists between the two spline shafts produces friction torque, which affects the torque transmitted through the shaft. Because the driving torque is changing with the shaft rotation, it will cause the rotation speed to change with the shaft rotation. The ripple of the rotation speed will lead to the friction torque change with the shaft rotation. Figure 6 shows the force and friction torque $T_{f_{2}}$ caused by the parallel misalignment $\Delta x$ I between the two spline shafts.

The torque transmitted by the torque transducer shaft $T_{2}$ is the drive torque minus the friction torque. When the parallel misalignment $\Delta x_{1}$ exists between the transmission shaft and the torque transducer shaft, the torque transmitted by the torque transducer shaft $T_{2}$ can be expressed as follows [34]:

$$
\begin{aligned}
T_{2}= & \bar{T}+\sum_{n} T_{n} \cos \left(n \theta_{\text {out }}+\varnothing_{n}\right)-\mu_{2} k_{2} r_{2} \Delta x^{\prime} \\
& -\mu_{2} m_{2} r_{2} \sum_{n} \omega_{n}^{2}\left(L_{0}-\Delta x^{\prime} \cos \left(n \theta_{\text {out }}+\varnothing_{n}\right)\right) \cos \left(n \theta_{\text {out }}+\varnothing_{n}\right),
\end{aligned}
$$

where $\bar{T}$ and $T_{n}$ are the averaged torque and amplitude for the $n$th driving torque, respectively, $n$ is the harmonic orders of the output shaft, $\theta_{\text {out }}$ and $\varnothing_{n}$ are the position of the output shaft and phase offset for the $n$th driving torque, respectively, $\mu_{2}$ is the friction coefficient of the torque transducer shaft, $m_{2}$ is the mass of the torque transducer shaft, $r_{2}$ is the radius of the torque transducer shaft, $k_{2}$ is the coefficient of elasticity of the torque transducer shaft, $\Delta x^{\prime}$ is the parallel misalignment, $L_{0}$ is the equivalent meshing distance of each spline's teeth in good alignment condition, and $\omega_{n}$ is the amplitude for the $n$th rotation speed of the shaft.

When an angular misalignment $\Delta \theta^{\prime}$ exists between the transmission shaft and the torque transducer shaft, the meshing of the spline shaft and spline sleeve is uneven. The friction torque $T_{f 2}^{\prime}$ caused by the uneven meshing of the spline shaft and spline sleeve and the sliding between the spline sleeve and the spline shaft is generated. Therefore, the angular misalignment $\Delta \theta^{\prime}$ that exists between the two spline shafts produces friction torque, which affects the torque transmitted through the shaft. Because the driving torque is changed with the shaft rotation, it will cause the rotation speed to change with the shaft rotation. The ripple of the rotation speed will lead to the friction torque change with the shaft rotation. Figure 7 shows the force and friction torque $T_{f 2}^{\prime}$ caused by the angular misalignment $\Delta \theta^{\prime}$ between the two spline shafts.

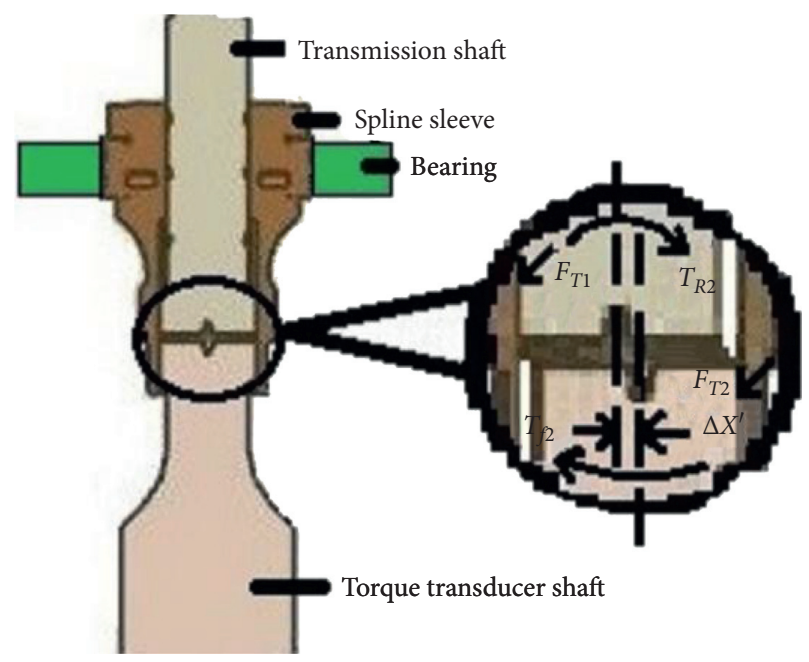

Figure 6: The force and the friction torque caused by the parallel misalignment of spline coupling.

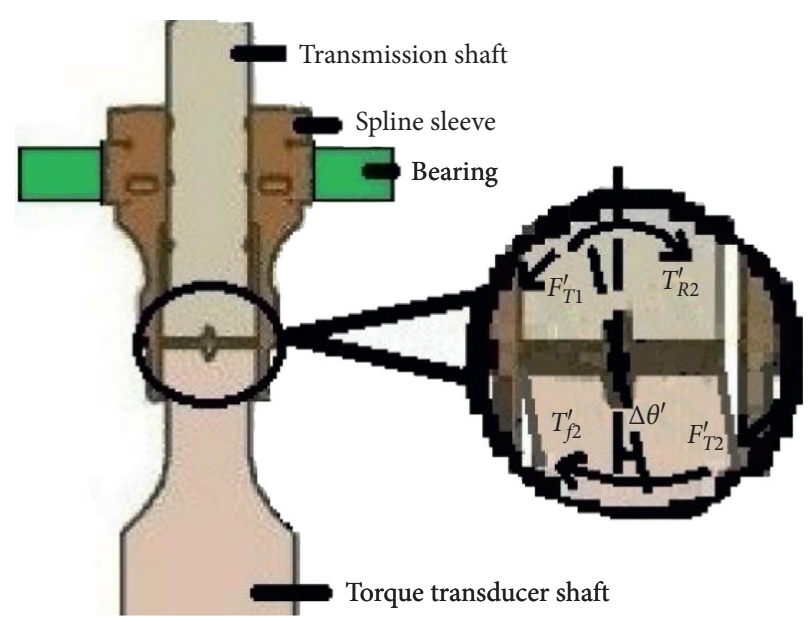

FIgURE 7: The force and the friction torque caused by the angular misalignment of spline coupling.

The torque transmitted by the torque transducer shaft $T_{2}^{\prime}$ is the drive torque minus the friction torque. When the angular misalignment $\Delta \theta^{\prime}$ exists between the transmission shaft and the torque transducer shaft, the torque transmitted by the torque transducer shaft $T_{2}^{\prime}$ can be expressed as follows [35]:

$$
\begin{aligned}
T_{2}^{\prime}= & \bar{T}+\sum_{n} T_{n} \cos \left(n \theta_{\text {out }}+\varnothing_{n}\right)-\mu_{2} k_{2} L_{2} \sin \left(\triangle \theta^{\prime}\right) \\
& -\mu_{2} k_{2} L_{2} \sin \left(\triangle \theta^{\prime}\right) \\
& -\mu_{2} m_{2} r_{2} \sum_{n} \omega_{n}^{2}\left(L_{0}-L_{2} \tan \triangle \theta^{\prime} \cos \left(n \theta_{\text {out }}+\varnothing_{n}\right)\right) \cos \left(n \theta_{\text {out }}+\varnothing_{n}\right),
\end{aligned}
$$


where $\bar{T}$ and $T_{n}$ are the averaged torque and amplitude for the $n$th driving torque, respectively, $n$ is the harmonic orders of the output shaft, $\theta_{\text {out }}$ and $\varnothing_{n}$ are the position of the output shaft and phase offset for the $n$th driving torque, respectively, $\mu_{2}$ is the friction coefficient of the torque transducer shaft, $m_{2}$ is the eccentric mass of the torque transducer shaft, $r_{2}$ is the radius of the torque transducer shaft, $k_{2}$ is the coefficient of elasticity of the torque transducer shaft, $\Delta \theta^{\prime}$ is the angular misalignment, $L_{0}$ is the equivalent meshing distance of each spline's teeth in good alignment condition, $\omega_{n}$ is the amplitude for the $n$th rotation speed of the shaft, and $L_{2}$ is the length of the torque transducer shaft.

\section{Experimental Results of Diagnosing the Misalignment Using the Fast Fourier Transform and Power Spectrum Analysis of the Torque Signal}

According to the analysis proposed in the above section, it can be seen that the amplitude of torque ripple of the shaft is linearly related to the misalignment of the flexible coupling and the spline coupling and is quadratically related to the rotation speed of the shaft when the parallel or angular misalignment exists in the shaft. Moreover, the frequency of the torque ripple is the same as the frequency of the rotation speed. So, the amplitude and frequency of torque ripple can be used to diagnose the misalignment of the MMIS and MMOS. Experiments have been done to test the capability of detecting the misalignment of the flexible coupling and the spline coupling of the MMIS and MMOS using the measurement and analysis of torque ripple. The Fast Fourier Transform and power spectrum analysis of torque are used to obtain the amplitude and frequency of torque ripple. The singular value decomposition (SVD) filtering stage is included in the data processing to obtain the amplitude and frequency of torque ripple. SVD filtering algorithm is to decompose the signal into a series of pure signal subspace and noise subspace, which are corresponding to the singular matrix. In the singular matrix, the larger singular value corresponds to the pure signal, and the smaller singular value corresponds to the noise signal. Therefore, after determining the reasonable effective rank of the singular matrix, the singular values with the same number of effective ranks are taken from the large to the small, and the remaining values are set to zero. Then the matrix estimation of pure signal is obtained by inverse operation of SVD, and the final signal after singular value denoising is obtained by inverse reconstruction of phase space. In signal processing, SVD filtering stage, which has excellent invariance and stability, is mainly used for the extraction of periodic components and the denoising of signals. By using the maximum salient point of singular value difference spectrum to accurately judge the order of noise reduction, the interference of noise components in the signal can be effectively eliminated; and the main components of fault information can be retained.
The structure of the MMIS and MMOS is introduced in the second section, as shown in Figure 1. In the experiment, the motor drives the transmission shaft coupled to the torque transducer shaft. That means the test mode conversion components make the shaft of the MMIS or MMOS under driven conditions. The angular misalignment conditions and radial misalignment conditions are controlled by adjusting the radial and angular displacements of the conversion components and the transmission shaft when installing the shaft of MMIS or MMOS, as shown in Figure 8. The torque transducers in MMIS and MMOS are used to test the torque ripple. The diagrammatic sketch of the experimental device (MMIS) is shown in Figure 9.

As an example, the details of adjusting the radial misalignment in the experiment are shown as follows: (1) Put the hollow cylinder in the center of the turntable. (2) Put the disk support on the hollow cylinder. (3) Adjust the radial misalignment between the torque transducer shaft and the disk support using the dial gauge. (4) Adjust the radial misalignment between the disk supports and the hollow cylinder using the dial gauge and install the disk support on the hollow cylinder. (5) Adjust the radial misalignment between the transmission shaft and the hollow cylinder using the dial gauge and install the transmission shaft on the disk support.

The torque transducers in the instrument are produced by the HBM company. The model of the torque transducer is T40B. The measuring range of the torque transducer in the MMIS is $0-50 \mathrm{Nm}$, and its measuring accuracy is $0.1 \%$ in full scale. The measuring range of the torque transducer in MMOS is $0-2000 \mathrm{Nm}$, and its measuring accuracy is $0.05 \%$ in full scale. As the best product of torque transducer of the world, the maximum allowable radial misalignment of T40B can reach about $2 \mathrm{~mm}$. But, when the radial misalignments reach more than $100 \mu \mathrm{m}$, the radial misalignments will lead to torque oscillation. The servo motor and torque motor used in the MMIS and MMOS are made by the Modrol Electric Co., Ltd. The model of the servo motor is SMS15-42P2C, and the model of the torque motor is MDD310. The loading accuracies of the servo motor and torque motor are $0.1 \%$ in full scale. The load ranges of the servo motor and torque motor are $50 \mathrm{Nm}$ and $2000 \mathrm{Nm}$, respectively. A PXIe acquisition system produced by the NI company is used to collect the torque transducer's signals. The accuracy of torque signal acquisition of the PXIe acquisition system is $0.1 \%$ in full scale. The torque signal data is collected using LabVIEW ${ }^{\mathrm{TM}}$ software at a rate of $25 \mathrm{k}$ samples per second.

First of all, experiments of diagnosing the misalignment fault of the shaft are carried out for alignment, a $0.5 \mathrm{~mm}$ radial misalignment, and a $1.0 \mathrm{~mm}$ radial misalignment. The experiments are carried out under no load. Using the MMIS as an example, the rotation speed is increased by $200 \mathrm{rpm}$ every time from $0 \mathrm{rpm}$ to $800 \mathrm{rpm}$. The torque transducer tests the transmitted torque of the MMIS under different rotation speeds. The ripple of the transmitted torque under different rotation speeds is analyzed using the Fast Fourier Transform and power spectrum analysis after SVD filtering. 


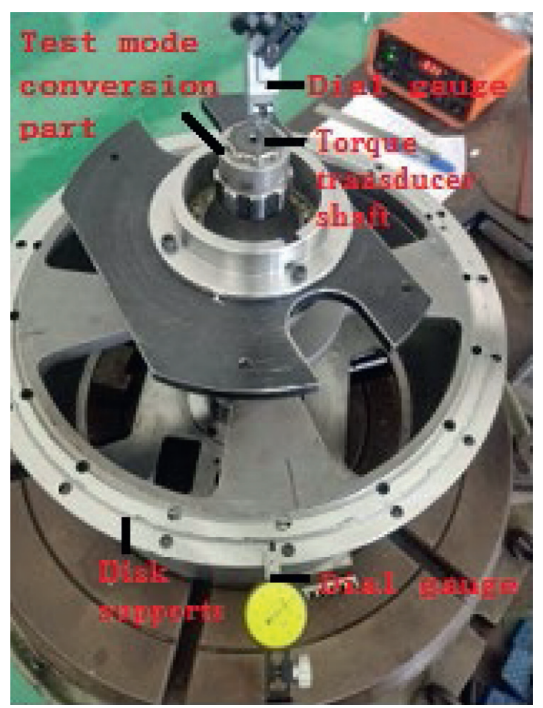

(a)

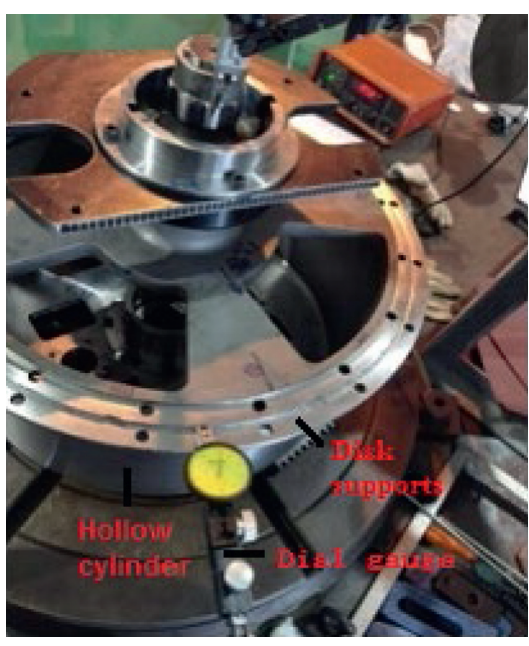

(b)

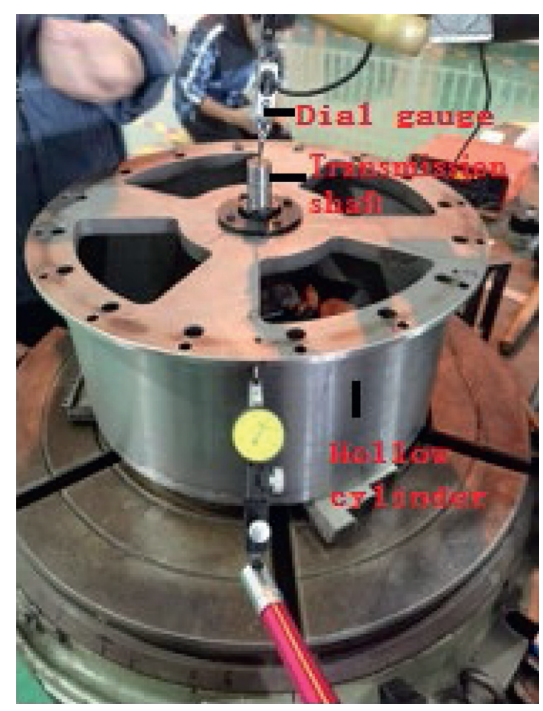

(c)

Figure 8: Adjusting the angular misalignment conditions and radial misalignment conditions of the shaft. (a) Adjusting the radial and angular misalignment of the torque transducer shaft; (b) adjusting the misalignment between the disk supports and the hollow cylinder; (c) adjusting the radial and angular misalignment of the transmission shaft.

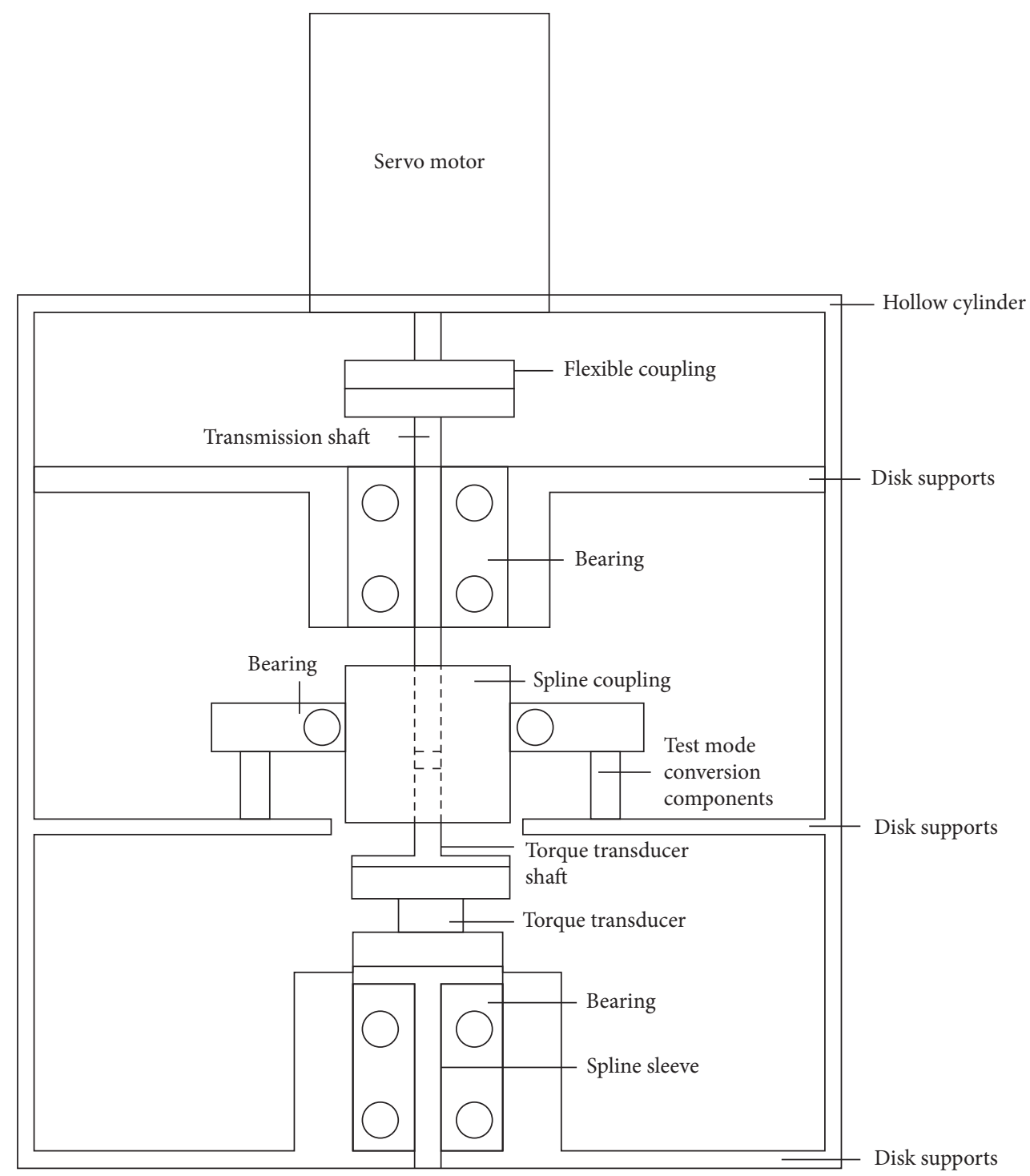

Figure 9: The diagrammatic sketch of the experimental device (MMIS). 


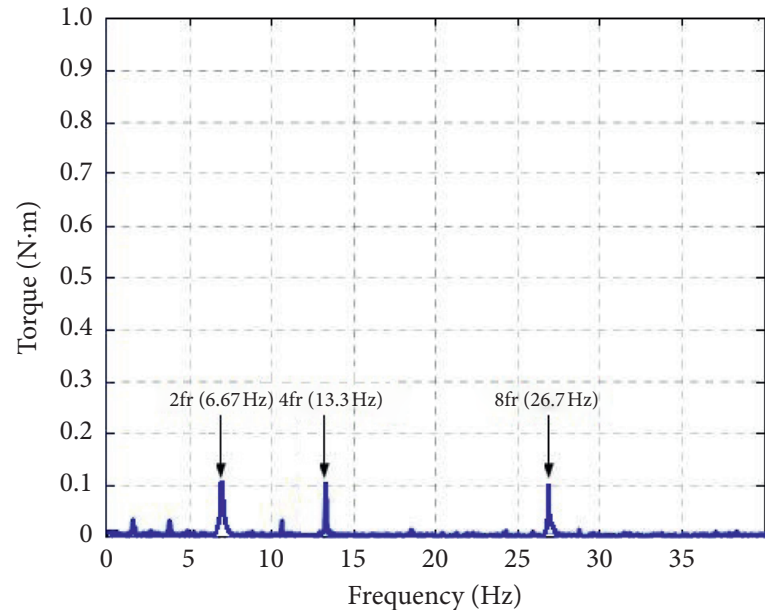

(a)

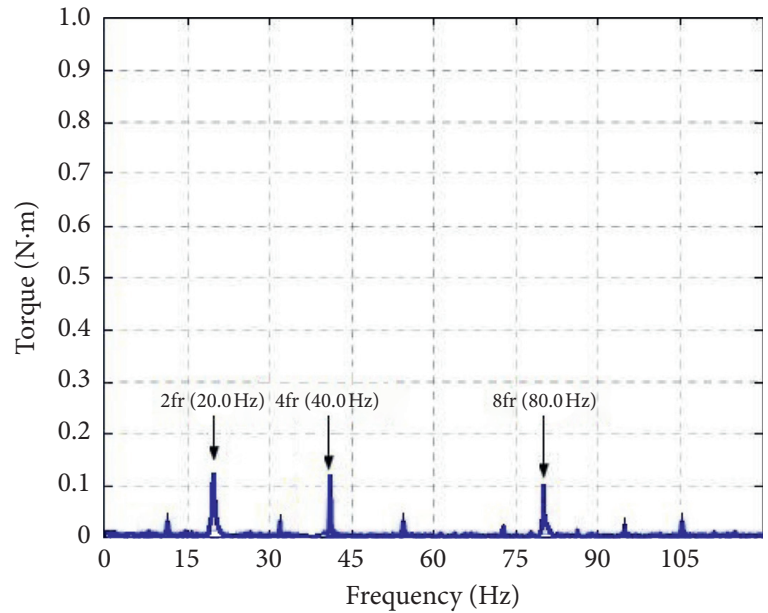

(c)

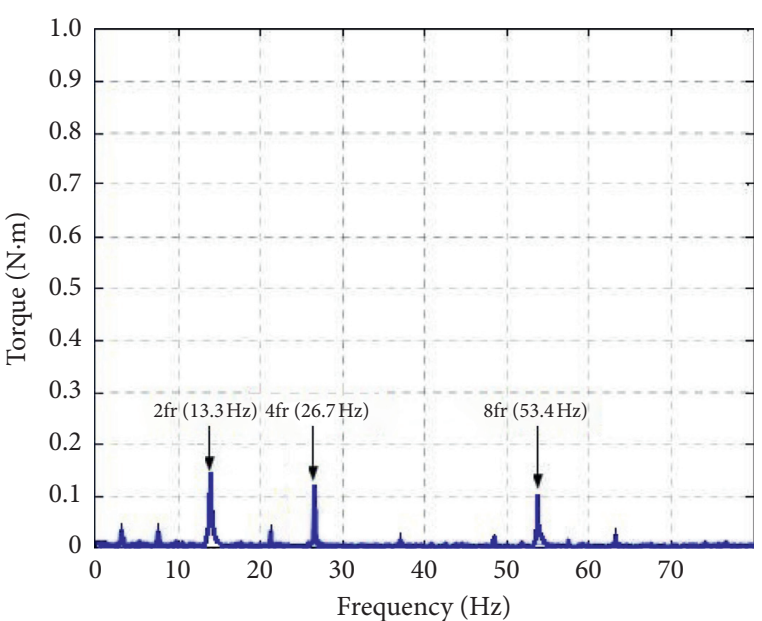

(b)

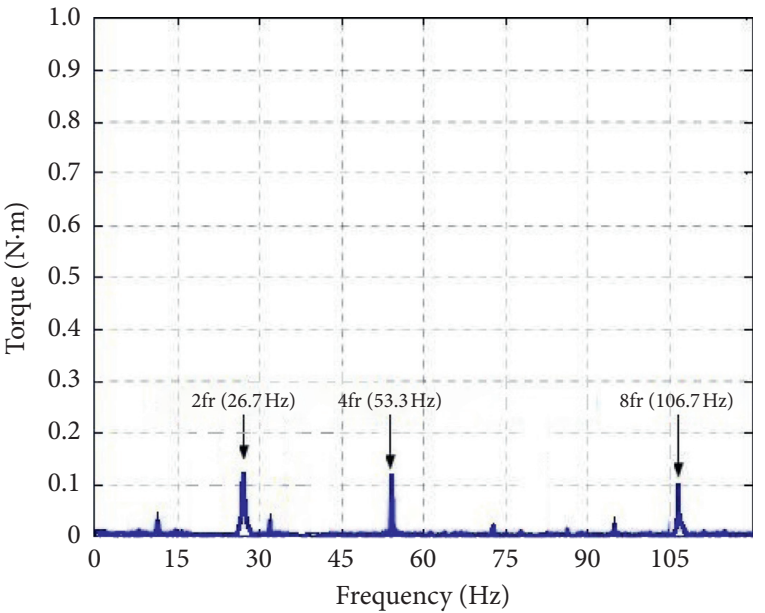

(d)

FIGURE 10: The frequency spectrum of the transmitted torque for shaft alignment under different rotation speeds. (a) $200 \mathrm{rpm}$; (b) $400 \mathrm{rpm}$; (c) $600 \mathrm{rpm}$; (d) $800 \mathrm{rpm}$.

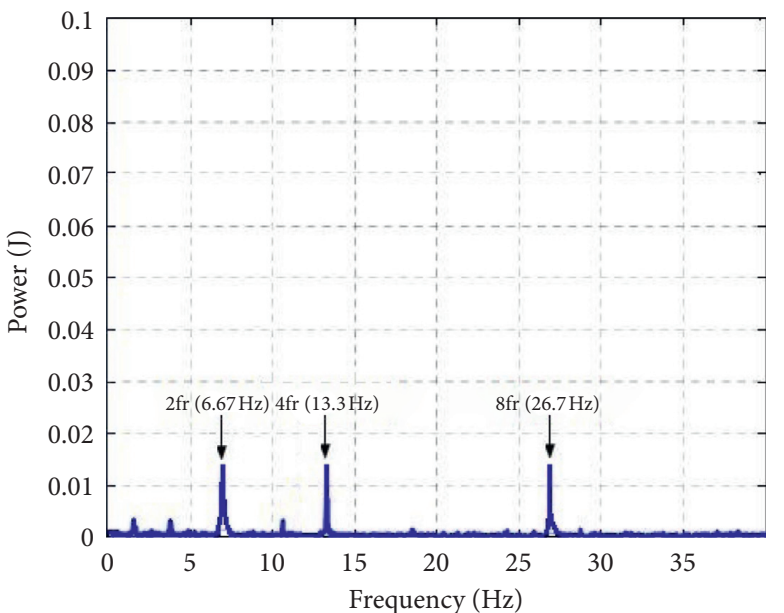

(a)

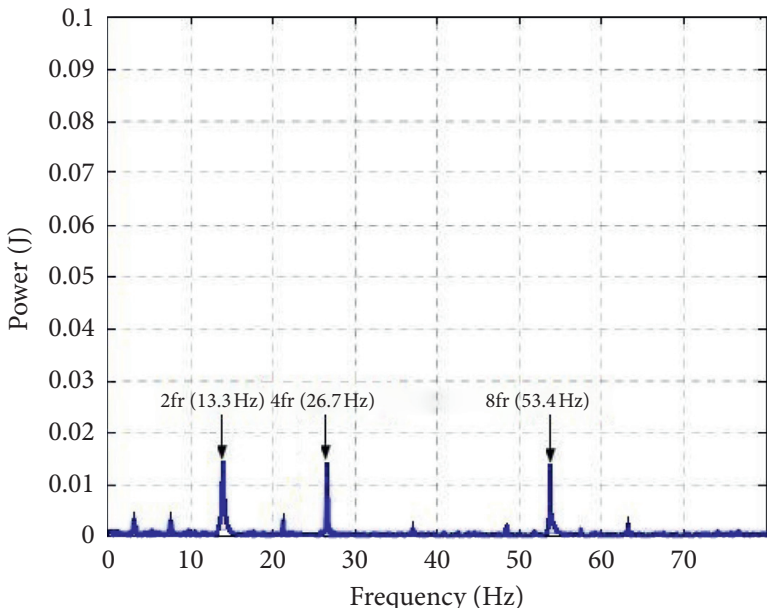

(b)

Figure 11: Continued. 


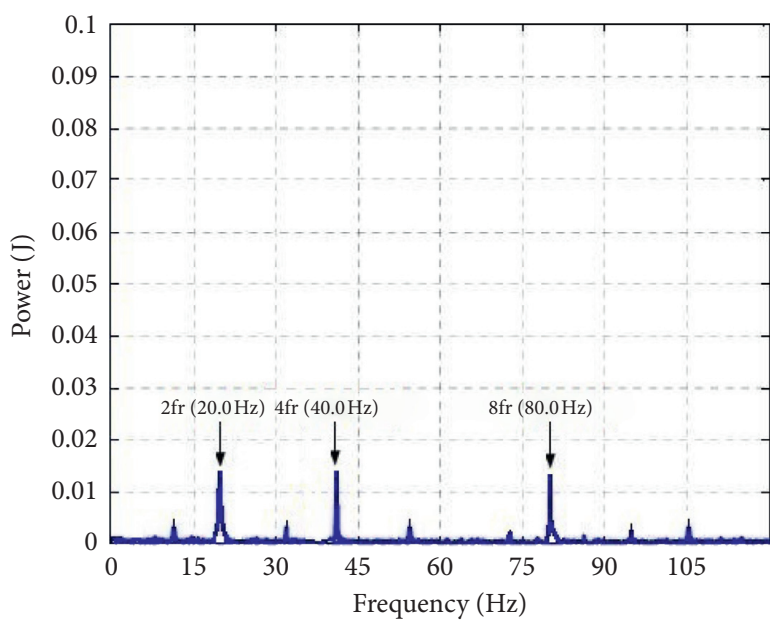

(c)

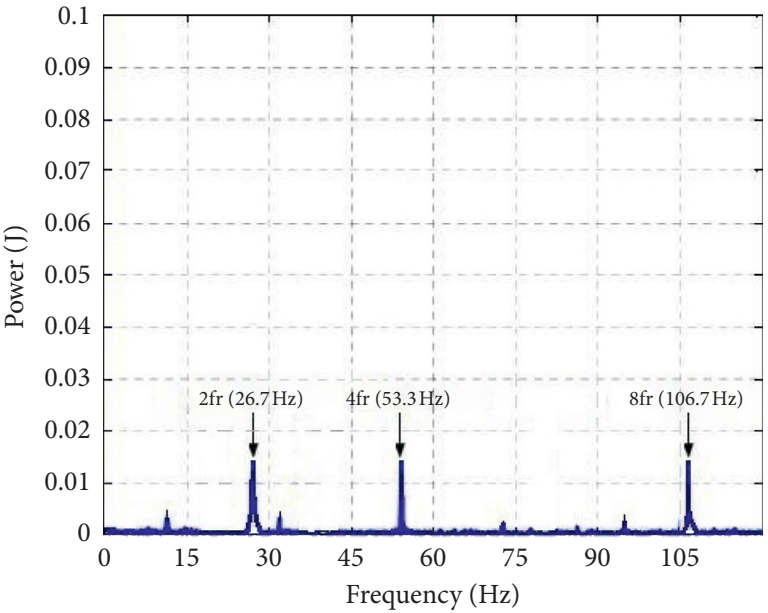

(d)

FIGURE 11: The power spectrum of the transmitted torque for shaft alignment under different rotation speeds. (a) $200 \mathrm{rpm}$; (b) $400 \mathrm{rpm}$; (c) $600 \mathrm{rpm}$; (d) $800 \mathrm{rpm}$.

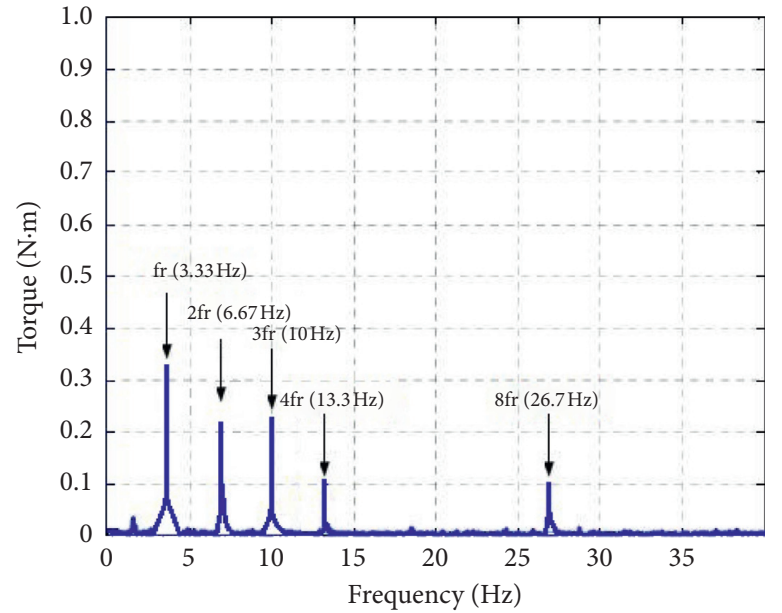

(a)

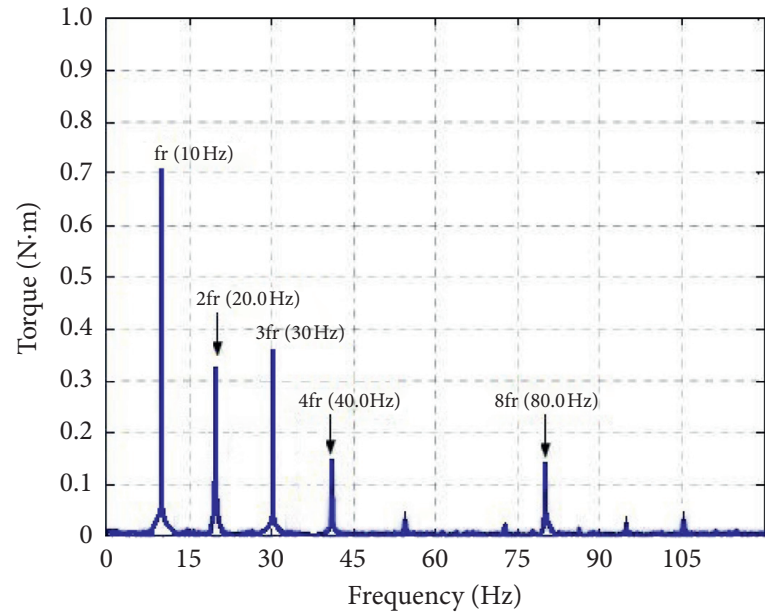

(c)

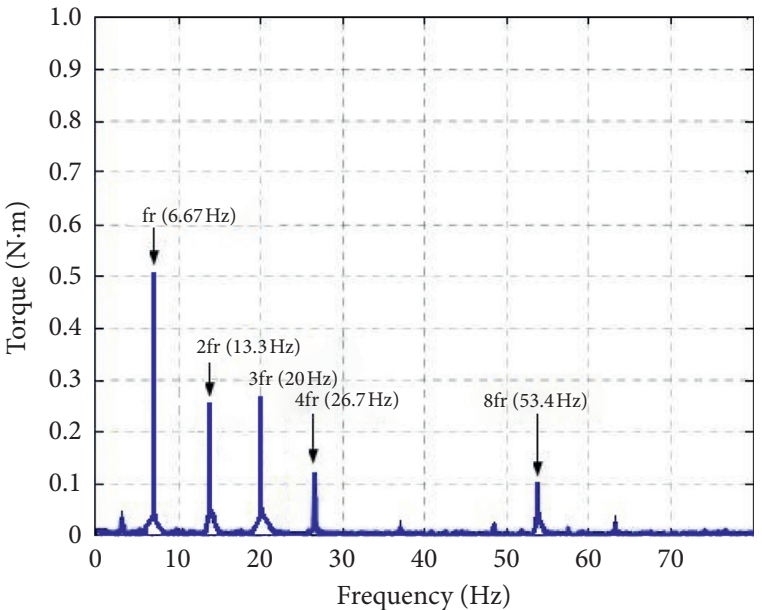

(b)

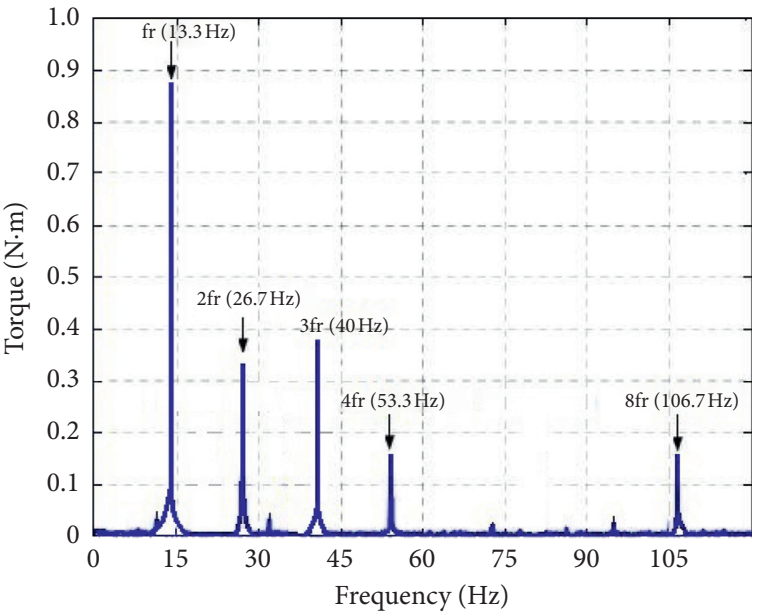

(d)

FigURE 12: The frequency spectrum of the transmitted torque for $0.5 \mathrm{~mm}$ radial misalignment under different rotation speeds. (a) $200 \mathrm{rpm}$; (b) $400 \mathrm{rpm}$; (c) $600 \mathrm{rpm}$; (d) $800 \mathrm{rpm}$. 


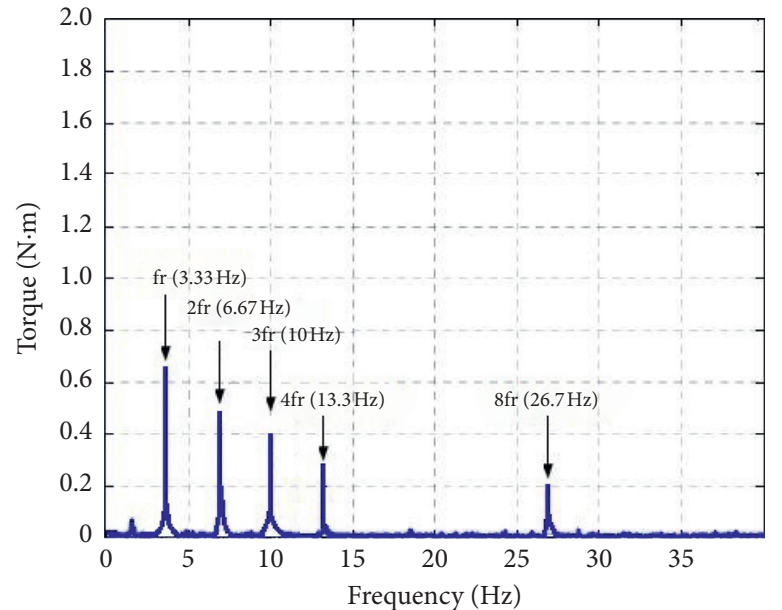

(a)

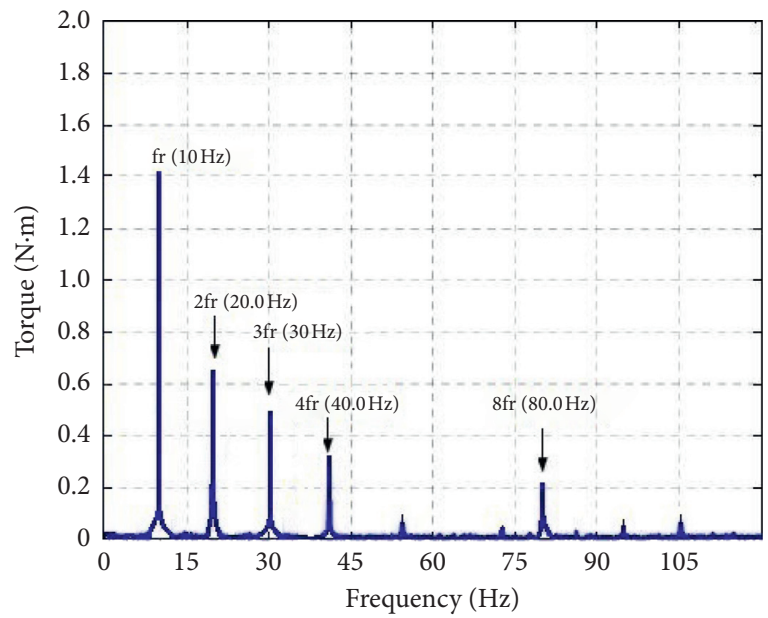

(c)

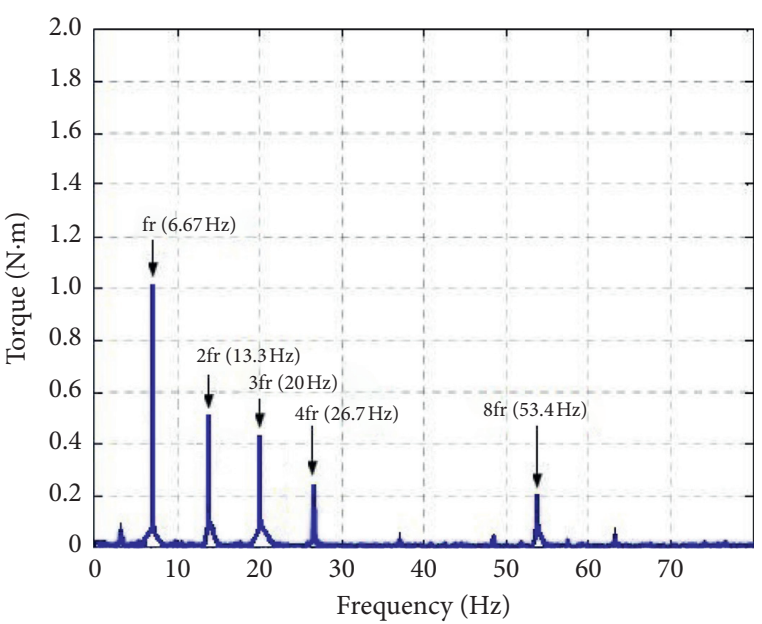

(b)

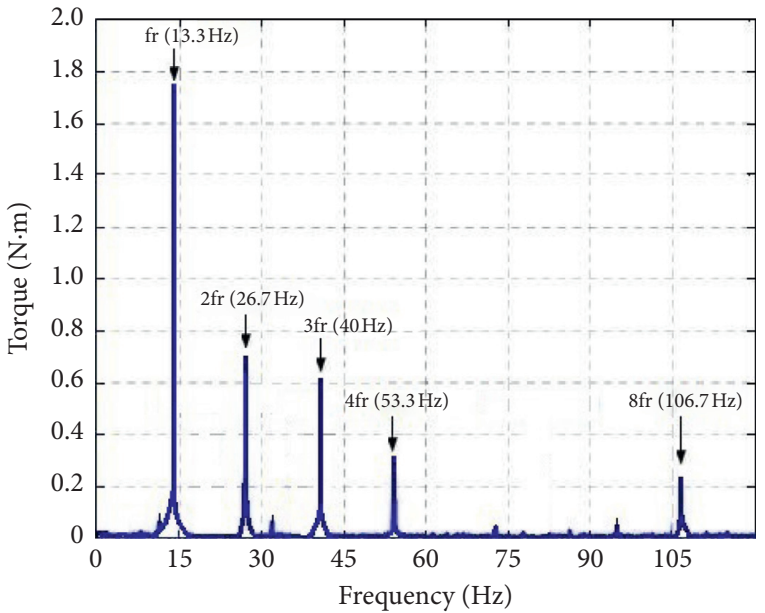

(d)

FIGURE 13: The frequency spectrum of the transmitted torque for $1.0 \mathrm{~mm}$ radial misalignment under different rotation speeds. (a) $200 \mathrm{rpm}$; (b) $400 \mathrm{rpm}$; (c) $600 \mathrm{rpm}$; (d) $800 \mathrm{rpm}$.

Figure 10 shows the frequency spectrum of the transmitted torque under different rotation speeds for the case of shaft alignment. For such conditions, the rotational frequency, when the rotation speed is increased by $200 \mathrm{rpm}$ every time from $0 \mathrm{rpm}$ to $800 \mathrm{rpm}$, is increased by $3.33 \mathrm{~Hz}$ every time from $0 \mathrm{~Hz}$ to $13.33 \mathrm{~Hz}$. The figure showing the absence of components at the rotation frequency (fr), which is shown in the third section, is able to detect fault misalignment. The additional components of twice the rotational frequency ( $2 \mathrm{fr}$ ), fourth of the rotational frequency (4fr), and even eighth of the rotational frequency (8fr) are also observed in Figure 10. This component may be due to the cogging torque and electromagnetic torque ripple. Figure 11 shows the power spectrum of the transmitted torque under different rotation speeds for the case of shaft alignment. The powers of the additional components of twice the rotational frequency ( $2 \mathrm{fr}$ ), fourth of the rotational frequency (4fr), and even eighth of the rotational frequency (8fr) are all the same. This phenomenon means that all the torque ripple causes, such as the cogging torque and electromagnetic torque ripple, have the same effect on the torque ripple.

Figure 12 shows the frequency spectrum for $0.5 \mathrm{~mm}$ radial misalignment. Figure 13 shows the frequency spectrum for $1.0 \mathrm{~mm}$ radial misalignment. In this particular case, a component at the rotation frequency (fr) of greater amplitude can be observed. These components clearly show misalignment between the transmission shaft and the torque transducer shaft. Furthermore, it can be observed that the components of twice the rotational frequency ( $2 \mathrm{fr}$ ), fourth of the rotational frequency (4fr), and eighth of the rotational frequency (8fr) are higher than those for aligned shafts. Figure 14 shows the power spectrum of the transmitted torque under different rotation speeds for $0.5 \mathrm{~mm}$ radial misalignment. Figure 15 shows the power spectrum of the transmitted torque under different rotation speeds for $1.0 \mathrm{~mm}$ radial misalignment. The powers of the additional components of the rotational frequency (fr), twice the rotational frequency (2fr), fourth of the rotational frequency (4fr), and even eighth of the rotational frequency (8fr) are 


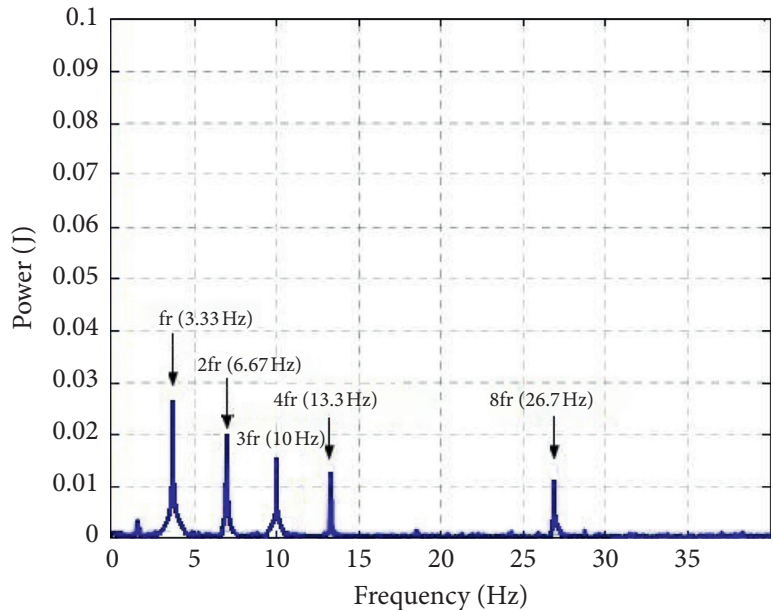

(a)

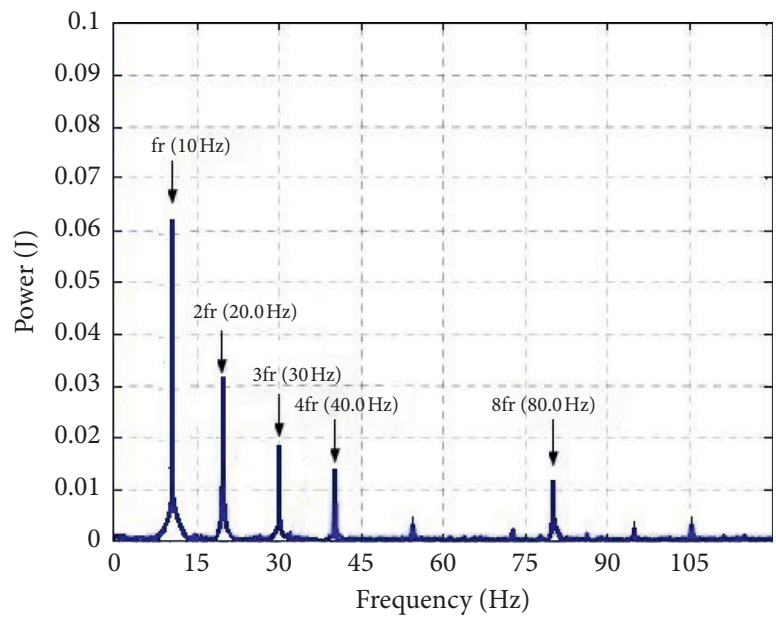

(c)

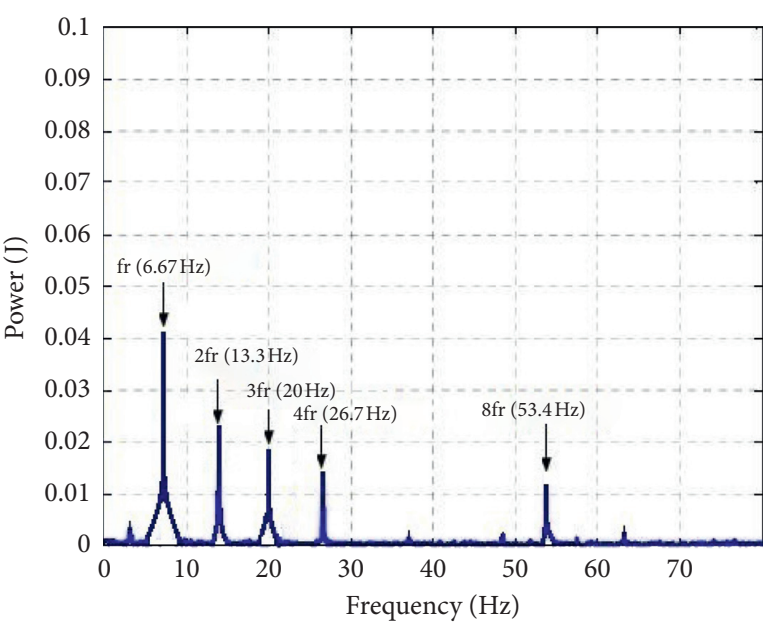

(b)

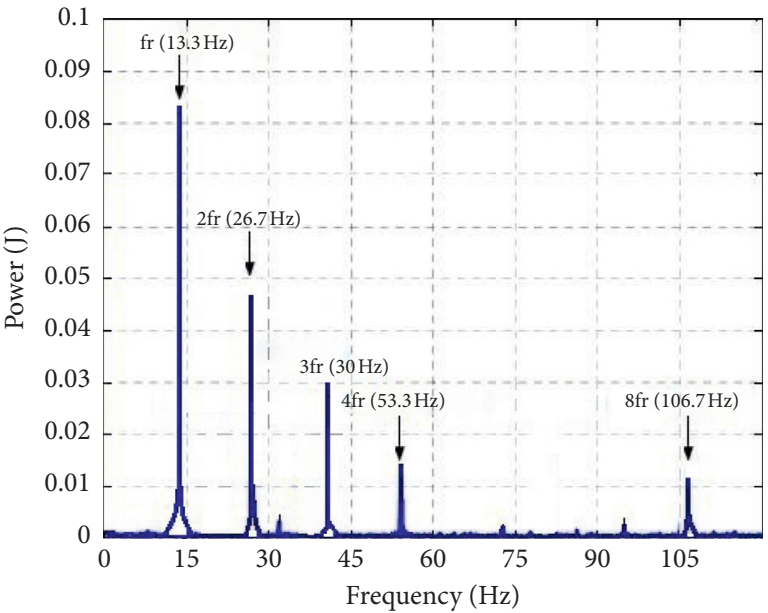

(d)

FIgURE 14: The power spectrum of the transmitted torque for $0.5 \mathrm{~mm}$ radial misalignment under different rotation speeds. (a) $200 \mathrm{rpm}$; (b) $400 \mathrm{rpm}$; (c) $600 \mathrm{rpm}$; (d) $800 \mathrm{rpm}$.

decreased with the increase of frequency. This phenomenon can be explained by the friction torque due to misalignment on shaft extension. Such torque increases the eccentricity which appears in the components at fr. After comparison, we can come to the conclusion that the component fr and the power of the additional components both allow diagnosing faults due to misalignment.

From Figures 10-15, it can be seen that the robustness and effects of the SVD filtering are so good that those unuseful signals of high frequency and low amplitudes have been filtered. That means only the main components of torque ripple, which can be used to diagnose the misalignment fault, left after SVD filtering.

In order to verify the diagnostic accuracy and robustness of the proposed method and the SVD filtering method, comparison experiments of diagnosing the misalignment fault of the shaft are carried out for alignment, a $0.125 \mathrm{~mm}$ radial misalignment, a $0.25 \mathrm{~mm}$ radial misalignment, a $0.375 \mathrm{~mm}$ radial misalignment, a $0.5 \mathrm{~mm}$ radial misalignment, a $0.625 \mathrm{~mm}$ radial misalignment, a $0.75 \mathrm{~mm}$ radial misalignment, a $0.875 \mathrm{~mm}$ radial misalignment, and a $1.0 \mathrm{~mm}$ radial misalignment. Figure 16 shows the amplitude of the components of torque ripple at $\mathrm{fr}, 2 \mathrm{fr}$, and $4 \mathrm{fr}$ for alignment, $0.125 \mathrm{~mm}$ radial misalignment, $0.25 \mathrm{~mm}$ radial misalignment, $0.375 \mathrm{~mm}$ radial misalignment, $0.5 \mathrm{~mm}$ radial misalignment, $0.625 \mathrm{~mm}$ radial misalignment, $0.75 \mathrm{~mm}$ radial misalignment, $0.875 \mathrm{~mm}$ radial misalignment, and $1.0 \mathrm{~mm}$ radial misalignment under the same rotation speeds. Figure 17 shows the amplitude of torque ripple at fr under different rotation speeds for alignment, $0.5 \mathrm{~mm}$ radial misalignment, and $1.0 \mathrm{~mm}$ radial misalignment, respectively. From the two figures, we can see that the amplitude of torque ripple of the shaft is linearly related to the radius misalignment and is quadratically related to the rotation speed of the shaft.

Then, comparison experiments of diagnosing the misalignment fault of the shaft are carried out for alignment, a $0.125^{\circ}$ angular misalignment, a $0.25^{\circ}$ angular misalignment, a $0.375^{\circ}$ angular misalignment, a $0.5^{\circ}$ angular misalignment, a $0.625^{\circ}$ angular misalignment, a $0.75^{\circ}$ 


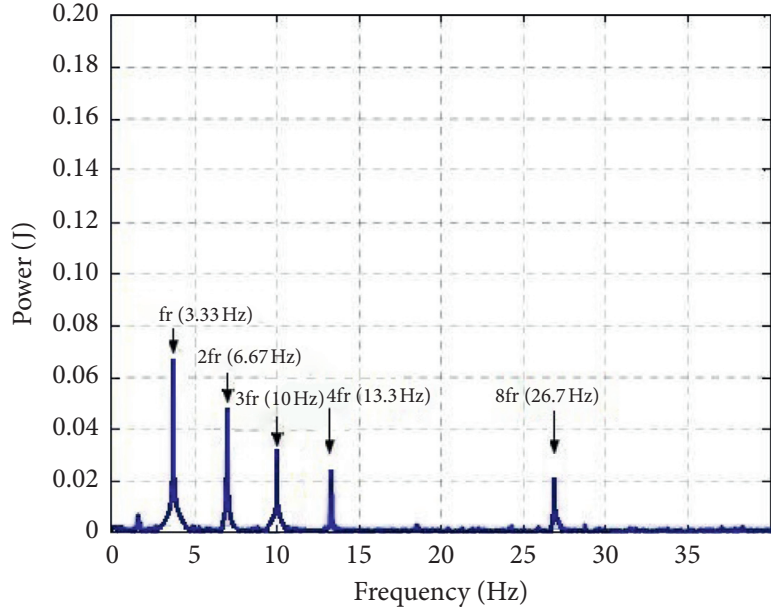

(a)

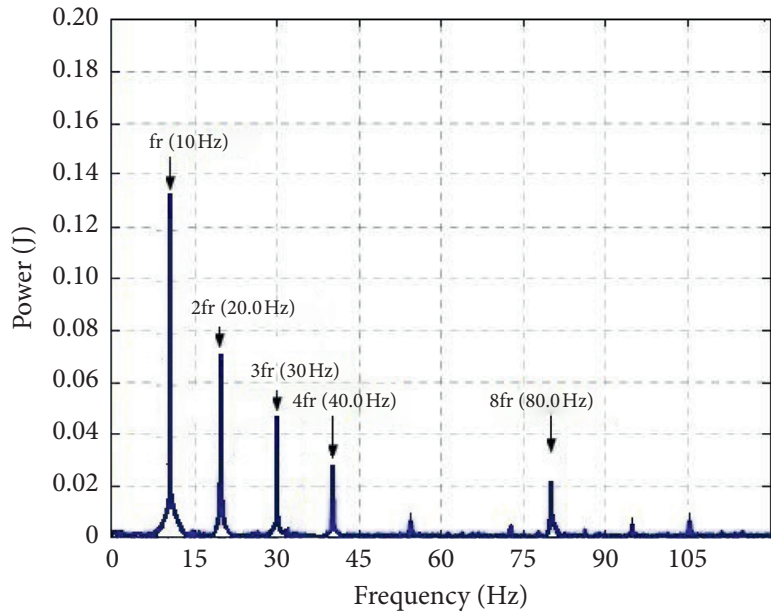

(c)

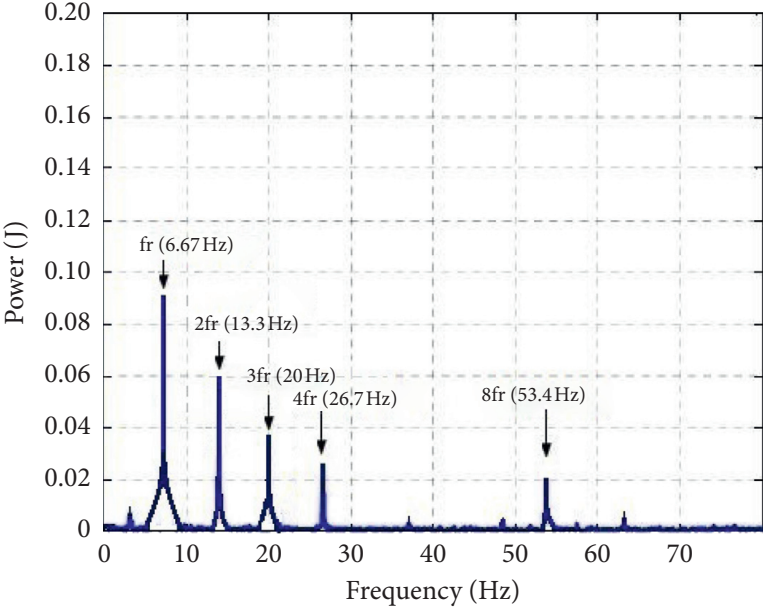

(b)

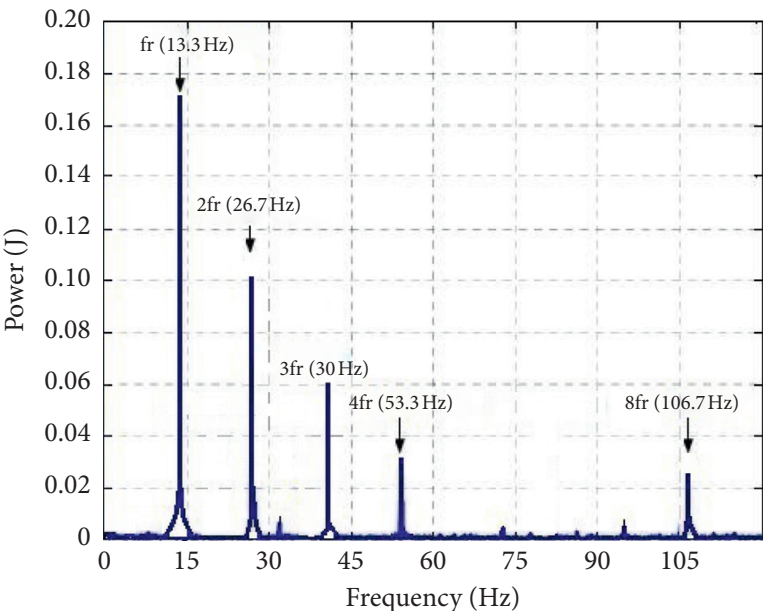

(d)

FIGURE 15: The power spectrum of the transmitted torque for $1.0 \mathrm{~mm}$ radial misalignment under different rotation speeds. (a) $200 \mathrm{rpm}$; (b) $400 \mathrm{rpm}$; (c) $600 \mathrm{rpm}$; (d) $800 \mathrm{rpm}$.

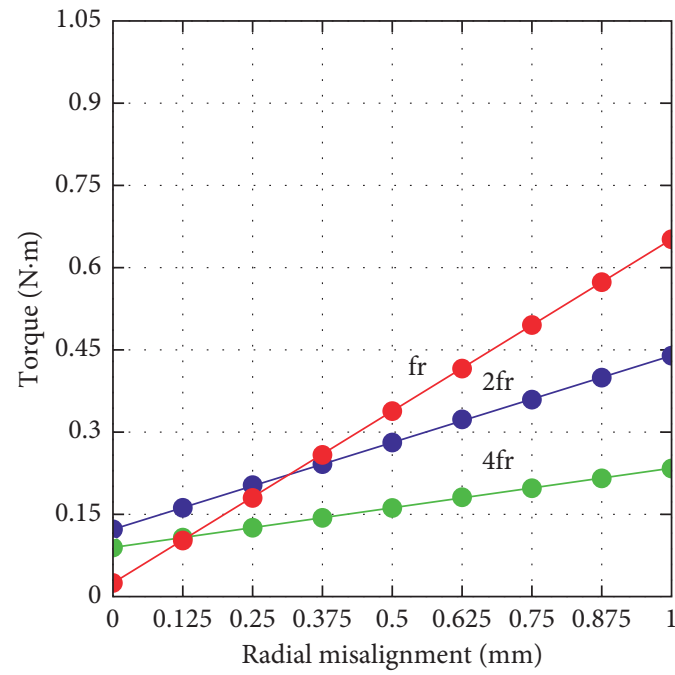

(a)

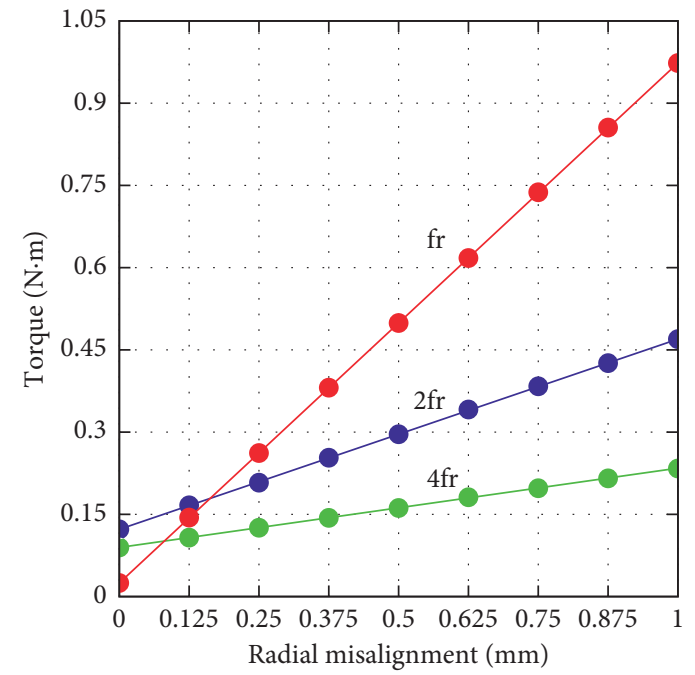

(b)

Figure 16: Continued. 


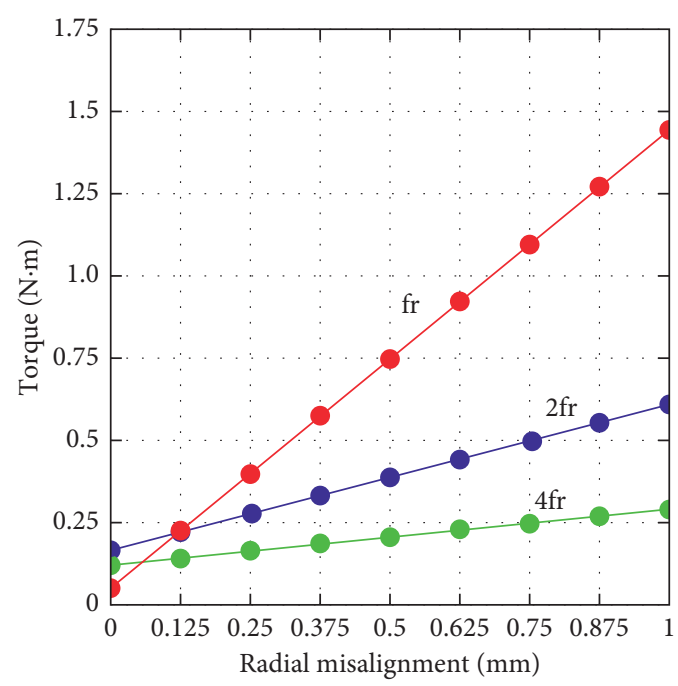

(c)

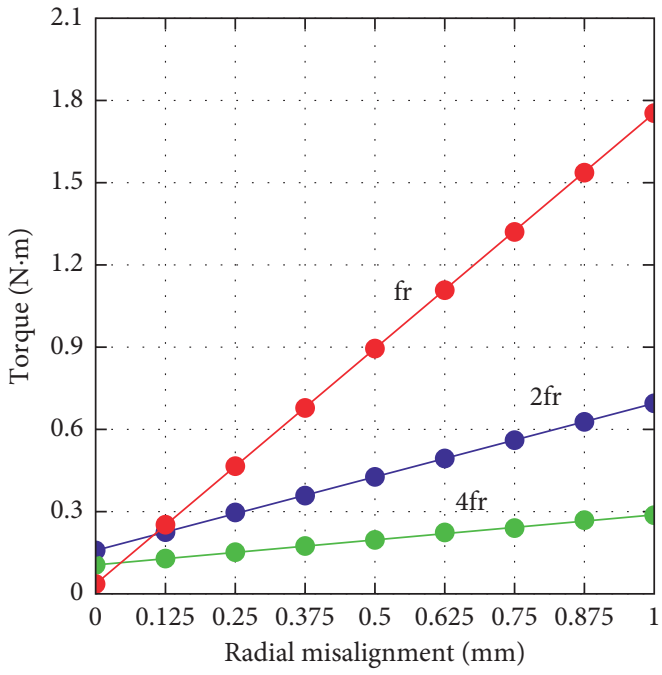

(d)

Figure 16: The amplitude of the torque ripple component at $\mathrm{fr}, 2 \mathrm{fr}$, and $4 \mathrm{fr}$ for alignment, $0.125 \mathrm{~mm}$ radial misalignment, $0.25 \mathrm{~mm}$ radial misalignment, $0.375 \mathrm{~mm}$ radial misalignment, $0.5 \mathrm{~mm}$ radial misalignment, $0.625 \mathrm{~mm}$ radial misalignment, $0.75 \mathrm{~mm}$ radial misalignment, $0.875 \mathrm{~mm}$ radial misalignment, and $1.0 \mathrm{~mm}$ radial misalignment under different rotation speeds. Red: fr; blue: $2 \mathrm{fr}$; green: $4 \mathrm{fr}$. (a) $200 \mathrm{rpm}$; (b) $400 \mathrm{rpm}$; (c) $600 \mathrm{rpm}$; (d) $800 \mathrm{rpm}$.

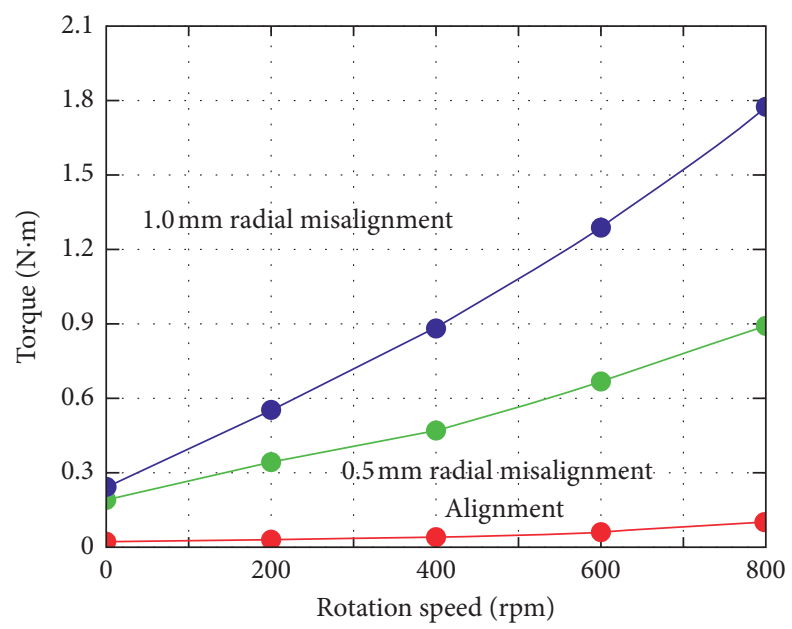

Figure 17: The amplitude of torque ripple at fr under different rotation speeds for alignment, $0.5 \mathrm{~mm}$ radial misalignment, and $1.0 \mathrm{~mm}$ radial misalignment. Red: alignment, blue: $0.5 \mathrm{~mm}$ radial misalignment, and green: $1.0 \mathrm{~mm}$ radial misalignment.

angular misalignment, a $0.875^{\circ}$ angular misalignment, and $1^{\circ}$ angular misalignment. The experiments are carried out under no load. Using the MMIS as an example, the rotation speed is increased by $200 \mathrm{rpm}$ every time from $0 \mathrm{rpm}$ to $800 \mathrm{rpm}$. The torque transducer tests the transmitted torque of the MMIS under different rotation speeds. Figure 18 shows the amplitudes of the component of torque ripple at $\mathrm{fr}, 2 \mathrm{fr}$, and $4 \mathrm{fr}$ for alignment, $0.125^{\circ}$ angular misalignment, $0.25^{\circ}$ angular misalignment, $0.375^{\circ}$ angular misalignment, $0.5^{\circ}$ angular misalignment, $0.625^{\circ}$ angular misalignment, $0.75^{\circ}$ angular misalignment, $0.875^{\circ}$ angular misalignment, and $1^{\circ}$ angular misalignment under the same rotation speeds. Figure 19 shows the amplitude of torque ripple at fr under different rotation speeds for alignment, $0.5^{\circ}$ angular misalignment, and $1^{\circ}$ angular misalignment, respectively. From the two figures, we can see that the amplitude of torque ripple of the shaft is linearly related to the angular misalignment and is quadratically related to the rotation speed of the shaft. 


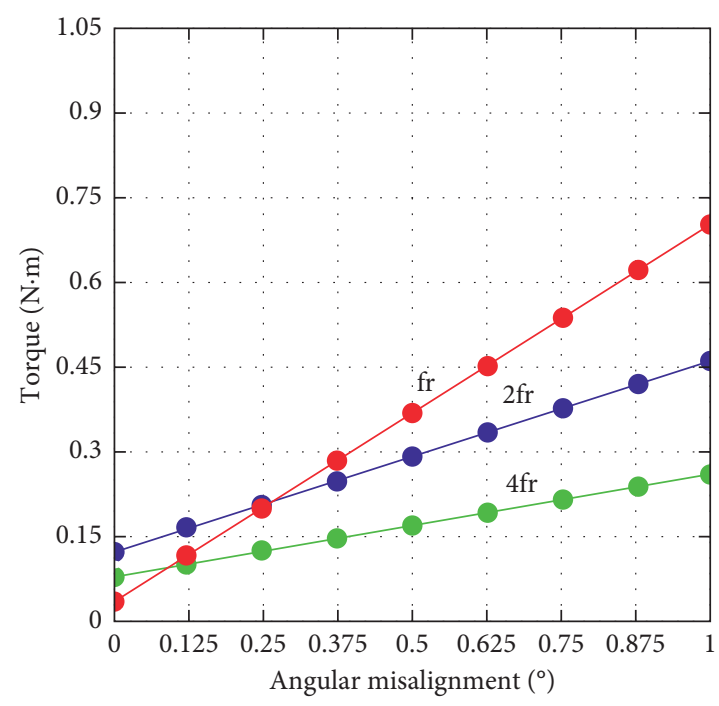

(a)

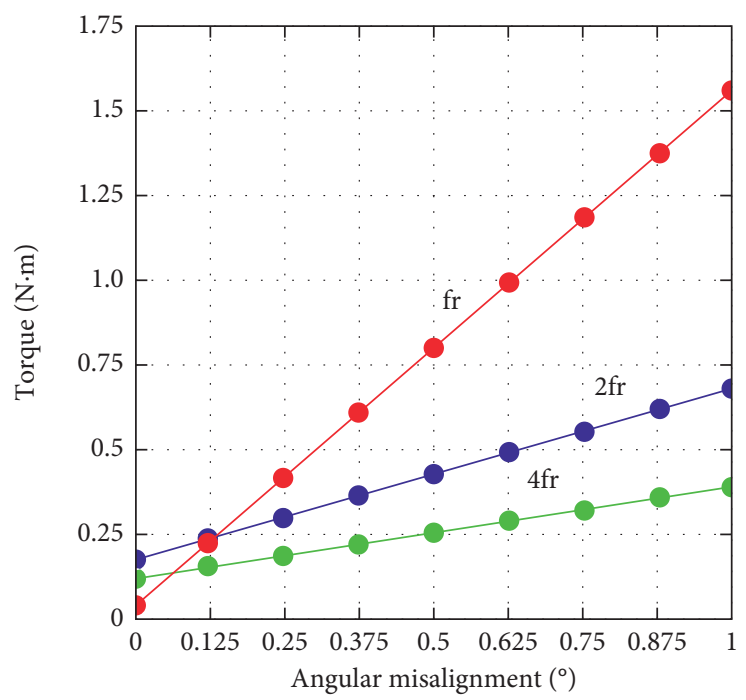

(c)

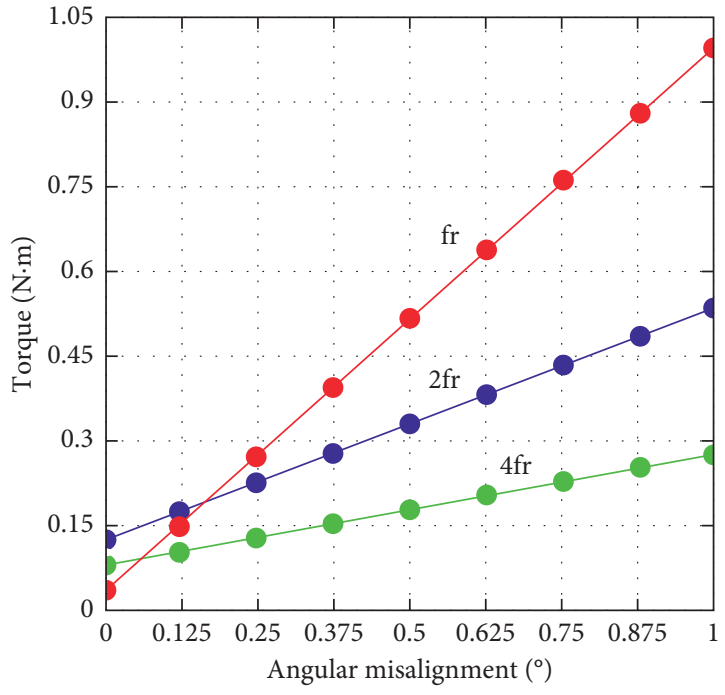

(b)

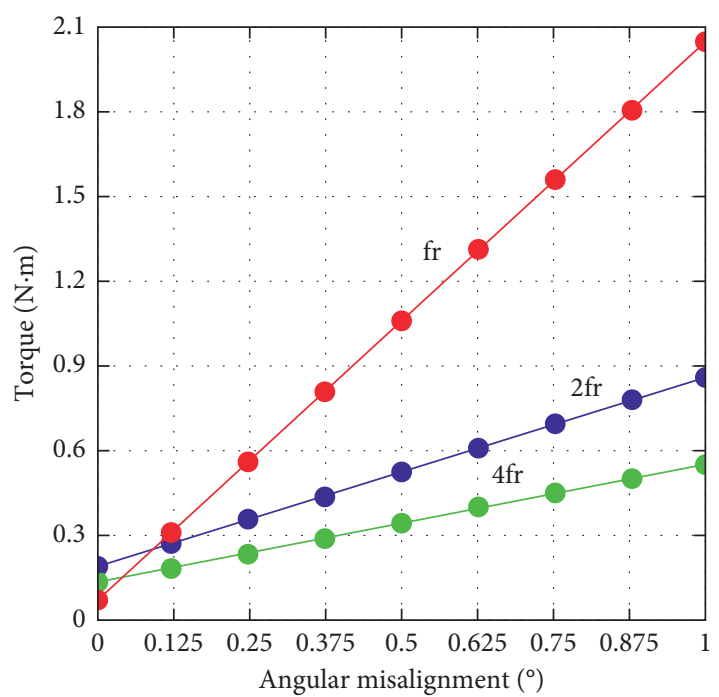

(d)

Figure 18: The amplitude of the torque ripple components at $\mathrm{fr}, 2 \mathrm{fr}$, and $4 \mathrm{fr}$ for alignment, $0.125^{\circ}$ angular misalignment, $0.25^{\circ}$ angular misalignment, $0.375^{\circ}$ angular misalignment, $0.5^{\circ}$ angular misalignment, $0.625^{\circ}$ angular misalignment, $0.75^{\circ}$ angular misalignment, $0.875^{\circ}$ angular misalignment, and $1^{\circ}$ angular misalignment under different rotation speeds. Red: fr; blue: $2 \mathrm{fr}$; green: $4 \mathrm{fr}$. (a) $200 \mathrm{rpm}$; (b) $400 \mathrm{rpm}$; (c) $600 \mathrm{rpm}$; (d) $800 \mathrm{rpm}$.

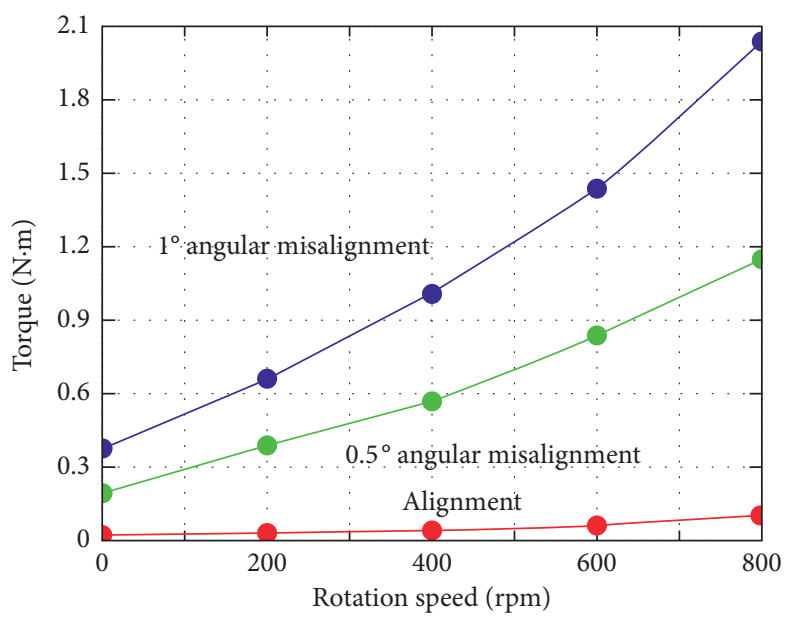

Figure 19: The amplitude of torque ripple at fr under different rotation speeds for alignment, $0.5^{\circ}$ angular misalignment, and $1^{\circ}$ angular misalignment. Red: alignment; blue: $0.5^{\circ}$ angular misalignment; green: $1^{\circ}$ angular misalignment. 


\section{Conclusions}

A method of using the torque transducer to diagnose the coupling misaligned fault of performance testing instruments of precision reducers is proposed in this paper. The causes of the coupling misaligned fault of the instrument and the relationship between the misalignment fault and torque ripple are analyzed. Experiments have been done to test the capability of detecting the coupling misalignment of the instrument using the measurement and analysis of torque ripple. From the analysis and experimental results presented in the third and fourth sections, we can see that the amplitude of torque ripple of the shaft is linearly related to the misalignment of the flexible coupling and the spline coupling and is quadratically related to the rotation speed of the shaft when the parallel or angular misalignment exists in the shaft. The amplitude and frequency of torque ripple can be used to diagnose the misalignment of the MMIS and MMOS. The frequency components of the torque ripple test by the torque transducer in the MMIS and MMOS allow identifying radial and angular misalignment when the increasing misalignment exists in the shaft connected by the flexible coupling and spline coupling.

Such a characteristic can be demonstrated with small levels of angular and radial misalignment faults. The components of the torque at $\mathrm{fr}$ and $2 \mathrm{fr}$ frequencies are suitable for detecting misalignment. Therefore, it is considered a more suitable fault indicator than that of the component at fr frequency. Moreover, the powers of the additional components of the rotational frequency ( $\mathrm{fr}$ ), twice the rotational frequency ( $2 \mathrm{fr}$ ), fourth of the rotational frequency (4fr), and even eighth of the rotational frequency (8fr) are decreased with the increase of frequency. This phenomenon can be explained by the friction torque due to misalignment on shaft extension. Such torque increases the eccentricity which appears in the components at fr. After comparison, we can come to the conclusion that the combination of components at the rotation frequency (fr) and the additional components can be used to diagnose faults due to coupling misalignment.

The method proposed in this paper is mainly about the diagnosis of static misalignment, caused by installation and the manufacturing tolerances. Those misaligned faults can lead to continuing periodical torque ripple. The frequency and the amplitude of torque ripple can be used to diagnose the misaligned fault. But when talking about the torque oscillation caused by the joint flexibility or backlash at joints, on one side, it is not periodical signal, and, on the other side, the amplitude of the torque oscillation will change with the rotation of shaft. So, the torque oscillation caused by the joint flexibility or backlash at joints will not influence the effect of the method proposed in this paper. The diagnosis of the joint flexibility or backlash at joints will be our research work in the future.

\section{Data Availability}

The data that support the findings of this study are available from the corresponding author upon reasonable request.

\section{Conflicts of Interest}

The authors declare that they have no conflicts of interest.

\section{Acknowledgments}

This research was financially supported by the National Key Research and Development Program of China (NKRDPC) (no. 2017YFF0108100).

\section{References}

[1] A.-D. Pham and H.-J. Ahn, "High precision reducers for industrial robots driving 4th industrial revolution: state of arts, analysis, design, performance evaluation and perspective," International Journal of Precision Engineering and Manufacturing-Green Technology, vol. 5, no. 4, pp. 519-533, 2018.

[2] X. Y. Chu, H. Xu, X. Wu, J. Tao, and G. Shao, "The method of selective assembly for the RV reducer based on genetic algorithm," Proceedings-Institution of Mechanical Engineers, Part C: Journal of Mechanical Engineering Science, vol. 232, no. 6, pp. 921-929, 2019.

[3] I. Makoto and N. Hiroyuki, "3rd international conference on advances in control and optimization of dynamical systems," IFAC Proceedings Volumes, vol. 47, no. 3, pp. 6831-6836, 2014.

[4] P. Peng and J. Wang, "NOSCNN: a robust method for fault diagnosis of RV reducer," Measurement, vol. 138, pp. 652658, 2019.

[5] J. Piotrowski, Shaft Alignment Handbook, CRC Press, Boca Raton, FL, USA, 3rd edition, 2006.

[6] R. Dhaouadi, F. H. Ghorbel, and P. S. Gandhi, "A new dynamic model of hysteresis in harmonic drives," IEEE Transactions on Industrial Electronics, vol. 50, no. 6, pp. 1165-1171, 2003.

[7] F. H. Ghorbel, P. S. Gandhi, and F. Alpeter, "On the kinematic error in harmonic drive gears," Journal of Mechanical Design, vol. 123, no. 1, pp. 90-97, 2001.

[8] P. S. Gandhi and F. H. Ghorbel, "Closed-loop compensation of kinematic error in harmonic drives for precision control applications," IEEE Transactions on Control Systems Technology, vol. 10, no. 6, pp. 759-768, 2002.

[9] S. H. Park, J. C. Park, S. W. Hwang, J. H. Kim, H. J. Park, and M. S. Lim, "Suppression of torque ripple caused by misalignment of the gearbox by using harmonic current injection method," IEEE/ASME Transactions on Mechatronics.vol. 99, pp. 1-10, 2020.

[10] J. Piotrowski, Shaft Alignment Handbook, Dekker, New York, NY, USA, 1986.

[11] M. Xu and R. D. Marangoni, "Vibration analysis of a motorflexible coupling-rotor system subject to misalignment and unbalance, part I: theoretical model and analysis," Journal of Sound and Vibration, vol. 176, no. 5, pp. 663-679, 1994.

[12] G. Xiang, Y. Han, J. Wang, K. Xiao, and J. Li, "A transient hydrodynamic lubrication comparative analysis for misaligned micro-grooved bearing considering axial reciprocating movement of shaft," Tribology International, vol. 132, pp. 11-23, 2019.

[13] W. Litwin, "Experimental research on water lubricated three layer sliding bearing with lubrication grooves in the upper part of the bush and its comparison with a rubber bearing," Tribology International, vol. 82, pp. 153-161, 2015.

[14] S. B. Shenoy and R. Pai, "Theoretical investigations on the performance of an externally adjustable fluid-film bearing 
including misalignment and turbulence effects," Tribology International, vol. 42, no. 7, pp. 1088-1100, 2009.

[15] J. M. Bossio, G. Bossio, and C. DeAngelo, "Angular misalignment in induction motors with flexible coupling," in Proceedings of IEEE Industrial Electronics Conference, pp. 1033-1038, IEEE, Porto, Portugal, November 2009.

[16] C. Verucchi, J. Bossio, G. Bossio, and G. Acosta, "Misalignment detection in induction motors with flexible coupling by means of estimated torque analysis and MCSA," Mechanical Systems and Signal Processing, vol. 80, pp. 570-581, 2016.

[17] K. Lu, Y. Jin, P. Huang, F. Zhang, H. Zhang, and C. Fu, “The applications of pod method in dual rotor-bearing systems with coupling misalignment," Mechanical Systems and Signal Processing, vol. 150, Article ID 107236, 2021.

[18] J. M. Bossio, C. H. De Angelo, and G. R. Bossio, "Self-organizing map approach for classification of mechanical and rotor faults on induction motors," Neural Computing \& Applications, vol. 23, no. 1, pp. 41-51, 2013.

[19] Y. Lei, Z. He, Y. Zi, and X. Chen, "New clustering algorithm-based fault diagnosis using compensation distance evaluation technique," Mechanical Systems and Signal Processing, vol. 22, no. 2, pp. 419-435, 2008.

[20] W. L. Du, J. F. Tao, Y. M. Li, and C. L. Liu, "Wavelet leaders multifractal features based fault diagnosis of rotating mechanism," Mechanical Systems and Signal Processing, vol. 43, no. 12, pp. 57-75, 2014.

[21] O. Janssens, V. Slavkovikj, B. Vervisch, K. Stockman, M. Loccufier, and S. Verstockt, "Convolutional neural network based fault detection for rotating machinery," Journal of Sound and Vibration, vol. 377, pp. 331-345, 2016.

[22] W. Zhou, B. Lu, T. G. Habetler, and R. G. Harley, "Incipient bearing fault detection via motor stator current noise cancellation using wiener filter," IEEE Transactions on Industry Applications, vol. 45, no. 4, pp. 1309-1317, 2009.

[23] A. D. Nembhard, J. K. Sinha, and A. Yunusa-Kaltungo, "Experimental observations in the shaft orbits of relatively flexible machines with different rotor related faults," Measurement, vol. 75, pp. 320-337, 2015.

[24] W. X. Yang and P. J. Tavner, "Empirical mode decomposition, an adaptive approach for interpreting shaft vibratory signals of large rotating machinery," Journal of Sound and Vibration, vol. 321, no. 3-5, pp. 1144-1170, 2009.

[25] A. K. Jalan and A. R. Mohanty, "Model-based fault diagnosis of a rotor-bearing system for misalignment and unbalance under steady-state condition," Journal of Sound and Vibration, vol. 327, no. 3-5, pp. 604-622, 2009.

[26] M. A. Khan, M. A. Shahid, S. A. Ahmed, S. Z. Khan, K. A. Khan, and S. A. Ali, "Gear misalignment diagnosis using statistical features of vibration and airborne sound spectrums," Measurement, vol. 145, pp. 419-435, 2019.

[27] L. Wang, F. Yun, S. Yao, and J. Liu, "Measurement method and pipe wall misalignment adjustment algorithm of the pipe butting machine," Measurement, vol. 94, pp. 873-882, 2016.

[28] T. H. Patel and A. K. Darpe, "Experimental investigations on vibration response of misaligned rotors," Mechanical Systems and Signal Processing, vol. 23, no. 7, pp. 2236-2252, 2009.

[29] J. H. Jung, B. C. Jeon, B. D. Youn, M. Kim, D. Kim, and Y. Kim, "Omnidirectional regeneration (ODR) of proximity sensor signals for robust diagnosis of journal bearing systems," Mechanical Systems and Signal Processing, vol. 90, pp. 189-207, 2017.

[30] J. Wang, Y. Peng, and W. Qiao, "Current-aided order tracking of vibration signals for bearing fault diagnosis of direct-drive wind turbines," IEEE Transactions on Industrial Electronics, vol. 63, no. 10, pp. 6336-6346, 2016.

[31] M. Chandra Sekhar Reddy and A. S. Sekhar, "Detection and monitoring of coupling misalignment in rotors using torque measurements," Measurement, vol. 61, pp. 111-122, 2015.

[32] S.-H. Park, J.-C. Park, S.-W. Hwang, J.-H. Kim, H.-J. Park, and M.-S. Lim, "Suppression of torque ripple caused by misalignment of the gearbox by using harmonic current injection method," IEEE, vol. 25, no. 4, pp. 1990-1999, 2020.

[33] X. Liu, D. Liang, J. Du, Y. Yu, X. Yang, and Z. Luo, "Effects analysis of misalignments on dynamic characteristics test for permanent magnet synchronous motor," in Proceedings of the 2014 17th International Conference on Electrical Machines and Systems (ICEMS), vol. 10, Hangzhou, China, 2014.

[34] J. Piotrowski, "Detecting misalignment on rotating machinery," in Shaft Alignment Handbook, pp. 35-87, CRC Press, Boca Raton, FL, USA, 3rd edition, 2007.

[35] C. Salomón, W. Santana, L. DaSilva, E. Bonaldi, L. DeOliveira, and J. DaSilva, "A stator flux synthesis approach for torque estimation of induction motors using a modified stator resistance considering the losses effect," in Proceedings of IEEE International Electric Machines \& Drives Conference, pp. 1369-1375, IEMDC, Chicago, IL, USA, May 2013. 